\title{
THE COMPLEXITY OF GENERALIZED SATISFIABILITY FOR LINEAR TEMPORAL LOGIC
}

MICHAEL BAULAND ${ }^{a}$, THOMAS SCHNEIDER $^{b}$, HENNING SCHNOOR $^{c}$, ILKA SCHNOOR $^{d}$, AND HERIBERT VOLLMER ${ }^{e}$

${ }^{a}$ Knipp GmbH, Martin-Schmeißer-Weg 9, 44227 Dortmund, Germany e-mail address: Michael.Bauland@knipp.de

${ }^{b}$ School of Computer Science, University of Manchester, Oxford Road, Manchester M13 9PL, UK e-mail address: schneider@cs.man.ac.uk

${ }^{c}$ Inst. für Informatik, Christian-Albrechts-Universität zu Kiel, 24098 Kiel, Germany e-mail address: schnoor@ti.informatik.uni-kiel.de

${ }^{d}$ Inst. für Theoretische Informatik, Universität zu Lübeck, Ratzeburger Allee 160, 23538 Lübeck, Germany

e-mail address: schnoor@tcs.uni-luebeck.de

${ }^{e}$ Inst. für Theoretische Informatik, Universität Hannover, Appelstr. 4, 30167 Hannover, Germany e-mail address: vollmer@thi.uni-hannover.de

\begin{abstract}
In a seminal paper from 1985, Sistla and Clarke showed that satisfiability for Linear Temporal Logic (LTL) is either NP-complete or PSPACE-complete, depending on the set of temporal operators used. If, in contrast, the set of propositional operators is restricted, the complexity may decrease. This paper undertakes a systematic study of satisfiability for LTL formulae over restricted sets of propositional and temporal operators. Since every propositional operator corresponds to a Boolean function, there exist infinitely many propositional operators. In order to systematically cover all possible sets of them, we use Post's lattice. With its help, we determine the computational complexity of LTL satisfiability for all combinations of temporal operators and all but two classes of propositional functions. Each of these infinitely many problems is shown to be either PSPACE-complete, NP-complete, or in P.
\end{abstract}

2000 ACM Subject Classification: F.4.1.

Key words and phrases: computational complexity, linear temporal logic, satisfiability.

* This article extends the conference contribution $\mathrm{BSS}^{+} 07$ with full proofs of all lemmata and theorems. Supported by the Postdoc Programme of the German Academic Exchange Service (DAAD).

Supported in part by DFG VO 630/6-1. 


\section{INTRODUCTION}

Linear Temporal Logic (LTL) was introduced by Pnueli in [Pnu77] as a formalism for reasoning about the properties and the behaviors of parallel programs and concurrent systems, and has widely been used for these purposes. Because of the need to perform reasoning tasks - such as deciding satisfiability, validity, or truth in a structure generated by binary relations - in an automated manner, their decidability and computational complexity is an important issue.

It is known that in the case of full LTL with the operators F (eventually), G (invariantly), $\mathrm{X}$ (next-time), $\mathrm{U}$ (until), and $\mathrm{S}$ (since), satisfiability and determination of truth are PSPACEcomplete SC85. Restricting the set of temporal operators leads to NP-completeness in some cases [SC85]. These results imply that reasoning with LTL is difficult in terms of computational complexity.

This raises the question under which restrictions the complexity of these problems decreases. Contrary to classical modal logics, there does not seem to be a natural way to modify the semantics of LTL and obtain decision problems with lower complexity. However, there are several possible constraints that can be posed on the syntax. One possibility is to restrict the set of temporal operators, which has been done exhaustively in [SC85, Mar04].

Another constraint is to allow only a certain "degree of propositionality" in the language, i.e., to restrict the set of allowed propositional operators. Every propositional operator represents a Boolean function-e.g., the operator $\wedge$ (and) corresponds to the binary function whose value is 1 if and only if both arguments have value 1 . There are infinitely many Boolean functions and hence an infinite number of propositional operators.

We will consider propositional restrictions in a systematic way, achieving a complete classification of the complexity of the reasoning problems for LTL. Not only will this reveal all cases in this framework where satisfiability is tractable. It will also provide a better insight into the sources of hardness by explicitly stating the combinations of temporal and propositional operators that lead to NP- or PSPACE-hard fragments. In addition, the "sources of hardness" will be identified whenever a proof technique is not transferable from an easy to a hard fragment.

Related work. The complexity of model-checking and satisfiability problems for several syntactic restrictions of LTL fragments has been determined in the literature: In [SC85, Mar04, temporal operators and the use of negation have been restricted; these fragments have been shown to be NP- or PSPACE-complete. In [DS02], temporal operators, their nesting, and the number of atomic propositions have been restricted; these fragments have been shown to be tractable or NP-complete. Furthermore, due to [CL93, DFR00], the restriction to Horn formulae does not decrease the complexity of satisfiability for LTL. As for related logics, the complexity of satisfiability has been shown in EES90 to be tractable or NP-complete for three fragments of CTL (computation tree logic) with temporal and propositional restrictions. In [Hal95, satisfiability for multimodal logics has been investigated systematically, bounding the depth of modal operators and the number of atomic propositions. In [Hem01], it was shown that satisfiability for modal logic over linear frames drops from NP-complete to tractable if propositional operators are restricted to conjunction and atomic negation.

The effect of propositional restrictions on the complexity of the satisfiability problem was first considered systematically by Lewis for the case of classical propositional logic in [Lew79]. He established a dichotomy - depending on the set of propositional operators, satisfiability is either NP-complete or decidable in polynomial time. In the case of modal 
propositional logic, a trichotomy has been achieved in [BHSS06]: modal satisfiability is PSPACE-complete, coNP-complete, or in P. That complete classification in terms of restrictions on the propositional operators follows the structure of Post's lattice of closed sets of Boolean functions [Pos41].

Our contribution. This paper analyzes the same systematic propositional restrictions for LTL, and combines them with restrictions on the temporal operators. Using Post's lattice, we examine the satisfiability problem for every possible fragment of LTL determined by an arbitrary set of propositional operators and any subset of the five temporal operators listed above. We determine the computational complexity of these problems, except for one case - where only propositional operators based on the binary xor function (and, perhaps, constants) are allowed. We show that all remaining cases are either PSPACE-complete, NP-complete, or in P.

It is not the aim of this paper to focus on particular propositional restrictions that are motivated by certain applications. We prefer to give a classification as complete as possible which allows to choose a fragment that is appropriate, in terms of expressivity and tractability, for any given application. Applications of syntactically restricted fragments of temporal logics can be found, for example, in the study of cryptographic protocols: In [Low08, Gavin Lowe restricts the application of negation and temporal operators to obtain practical verification algorithms.

Among our results, we exhibit cases with non-trivial tractability as well as the smallest possible sets of propositional and temporal operators that already lead to NP-completeness or PSPACE-completeness, respectively. Examples for the first group are cases in which only the unary not function, or only monotone functions are allowed, but there is no restriction on the temporal operators. As for the second group, if only the binary function $f$ with $f(x, y)=$ $(x \wedge \bar{y})$ is permitted, then satisfiability is NP-complete already in the case of propositional logic [Lew79]. Our results show that the presence of the same function $f$ separates the tractable languages from the NP-complete and PSPACE-complete ones, depending on the set of temporal operators used. According to this, minimal sets of temporal operators leading to PSPACE-completeness together with $f$ are, for example, $\{\mathrm{U}\}$ and $\{\mathrm{F}, \mathrm{X}\}$.

The technically most involved proof is that of PSPACE-hardness for the language with only the temporal operator $\mathbf{S}$ and the boolean operator $f$ (Theorem 3.3). The difficulty lies in simulating the quantifier tree of a Quantified Boolean Formula (QBF) in a linear structure.

Our results are summarized in Table 1. The first column contains the sets of propositional operators, with the terminology taken from Definition 2.2. The second column shows the classification of classical propositional logic as known from Lew79 and Coo71. The last line in column 3 and 4 is largely due to $[\mathrm{SC} 85$. All other entries are the main results of this paper. The only open case appears in the third line and is discussed in the Conclusion. Note that the case distinction also covers all clones which are not mentioned in the present paper.

\section{Preliminaries}

A Boolean function or Boolean operator is a function $f:\{0,1\}^{n} \rightarrow\{0,1\}$. We can identify an $n$-ary propositional connector $c$ with the $n$-ary Boolean operator $f$ defined by: $f\left(a_{1}, \ldots, a_{n}\right)=1$ if and only if the formula $c\left(x_{1}, \ldots, x_{n}\right)$ becomes true when assigning $a_{i}$ to $x_{i}$ for all $1 \leq i \leq n$. Additionally to propositional connectors we use the unary temporal 


\begin{tabular}{l|c|cc} 
set of temporal operators & $\emptyset$ & $\{\mathrm{F}\},\{\mathrm{G}\}$, & any other \\
set of propositional operators & & $\{\mathrm{F}, \mathrm{G}\},\{\mathrm{X}\}$ & combination \\
\hline all operators 1-reproducing or self-dual & trivial & trivial & trivial \\
only negation or all operators monotone & in P & in P & in P \\
all operators linear & in P & $?$ & $?$ \\
$x \wedge \neg y$ is expressible & NP-c. & NP-c. & PSPACE-c. \\
all Boolean functions & NP-c. & NP-c. & PSPACE-c.
\end{tabular}

Table 1: Complexity results for satisfiability. The entries "trivial" denote cases in which a given formula is always satisfiable. The abbreviation "c." stands for "complete." Question marks stand for open questions.

operators X (next-time), F (eventually), G (invariantly) and the binary temporal operators $\mathrm{U}$ (until), and $\mathrm{S}$ (since).

Let $B$ be a finite set of Boolean functions and $M$ be a set of temporal operators. A temporal $B$-formula over $M$ is a formula $\varphi$ that is built from variables, propositional connectors from $B$, and temporal operators from $M$. More formally, a temporal $B$-formula over $M$ is either a propositional variable or of the form $f\left(\varphi_{1}, \ldots, \varphi_{n}\right)$ or $g\left(\varphi_{1}, \ldots, \varphi_{m}\right)$, where $\varphi_{i}$ are temporal $B$-formulae over $M, f$ is an $n$-ary propositional operator from $B$ and $g$ is an $m$-ary temporal operator from $M$. In [SC85], complexity results for formulae using the temporal operators F, G, X (unary), and U, S (binary) were presented. We extend these results to temporal $B$-formulae over subsets of those temporal operators. The set of variables appearing in $\varphi$ is denoted by $V_{\varphi}$. If $M=\{\mathrm{X}, \mathrm{F}, \mathrm{G}, \mathrm{U}, \mathrm{S}\}$ we call $\varphi$ a temporal $B$-formula, and if $M=\emptyset$ we call $\varphi$ a propositional $B$-formula or simply a $B$-formula. The set of all temporal $B$-formulae over $M$ is denoted by $\mathrm{L}(M, B)$.

A model in linear temporal logic is a linear structure of states, which intuitively can be seen as different points of time, with propositional assignments. Formally a structure $S=(s, V, \xi)$ consists of an infinite sequence $s=\left(s_{i}\right)_{i \in \mathbb{N}}$ of distinct states, a set of variables $V$, and a function $\xi:\left\{s_{i} \mid i \in \mathbb{N}\right\} \rightarrow 2^{V}$ which induces a propositional assignment of $V$ for each state. be a structure and $\varphi$ a temporal $\{\wedge, \neg\}$-formula over $\{\mathrm{X}, \mathrm{U}, \mathrm{S}\}$ with variables from $V$. We define what it means that $S$ satisfies $\varphi$ in $s_{i}\left(S, s_{i} \vDash \varphi\right)$ : For a temporal $\{\wedge, \neg\}$ formula over $\{\mathrm{X}, \mathrm{U}, \mathrm{S}\}$ with variables from $V$ we define what it means that $S$ satisfies $\varphi$ in $s_{i}\left(S, s_{i} \vDash \varphi\right)$ : let $\varphi_{1}$ and $\varphi_{2}$ be temporal $\{\wedge, \neg\}$-formulae over $\{\mathrm{X}, \mathrm{U}, \mathrm{S}\}$ and $x \in V$ a variable.

$$
\begin{array}{lll}
S, s_{i} \vDash x & \text { if and only if } & x \in \xi\left(s_{i}\right), \\
S, s_{i} \vDash \varphi_{1} \wedge \varphi_{2} & \text { if and only if } & S, s_{i} \vDash \varphi_{1} \text { and } S, s_{i} \vDash \varphi_{2}, \\
S, s_{i} \vDash \neg \varphi_{1} & \text { if and only if } & S, s_{i} \not \models \varphi_{1}, \\
S, s_{i} \vDash \boldsymbol{X}_{1} & \text { if and only if } & S, s_{i+1} \vDash \varphi_{1}, \\
S, s_{i} \vDash \varphi_{1} \cup \varphi_{2} & \text { if and only if } & \text { there is a } k \geq i \text { such that } S, s_{k} \vDash \varphi_{2}, \\
& & \text { and for every } i \leq j<k, S, s_{j} \vDash \varphi_{1}, \\
S, s_{i} \vDash \varphi_{1} S \varphi_{2} & \text { if and only if } & \text { there is a } k \leq i \text { such that } S, s_{k} \vDash \varphi_{2}, \\
& & \text { and for every } k<j \leq i, S, s_{j} \vDash \varphi_{1} .
\end{array}
$$

The remaining temporal operators are interpreted as abbreviations: $\mathrm{F} \varphi=\operatorname{true} \mathrm{U} \varphi$ and $\mathrm{G} \varphi=\neg \mathrm{F} \neg \varphi$. Therefore and since every Boolean operator can be composed from $\wedge$ and 
$\neg$, the above definition generalizes to temporal $B$-formulae for arbitrary sets $B$ of Boolean operators.

A temporal $B$-formula $\varphi$ over $M$ is satisfiable if there exists a structure $S$ such that $S, s_{i} \vDash \varphi$ for some state $s_{i}$ from $S$. Furthermore, $\varphi$ is called valid if, for all structures $S$ and all states $s_{i}$ from $S$, it holds that $S, s_{i} \vDash \varphi$. We will consider the following problems: Let $B$ be a finite set of Boolean functions and $M$ a set of temporal operators. Then $\operatorname{SAT}(M, B)$ is the problem to decide whether a given temporal $B$-formula over $M$ is satisfiable. In the literature, another notion of satisfiability is sometimes considered, where we ask if a formula can be satisfied at the first state in a structure. It is easy to see that, in terms of computational complexity, this does not make a difference for our problems as long as the considered fragment does not contain the temporal operator S. For this paper, we only study the satisfiability problem as defined above.

Sistla and Clarke analyzed the satisfiability problem for temporal $\{\wedge, \vee, \neg\}$-formulae over some sets of temporal operators, see Theorem 2.1. Note that, due to de Morgan's laws, there is no significant difference between the sets $\{\wedge, \vee, \neg\}$ and $\{\wedge, \neg\}$ of Boolean operators. For convenience, we will therefore prefer the former denotation to the latter when stating results. Furthermore, the original proof of Theorem 2.1 explicitly uses the operator $\vee$.

Theorem 2.1 ([SC85]).

(1) $\operatorname{SAT}(\{\mathrm{F}\},\{\wedge, \vee, \neg\})$ is NP-complete.

(2) $\operatorname{SAT}(\{F, X\},\{\wedge, \vee, \neg\}), \operatorname{SAT}(\{U\},\{\wedge, \vee, \neg\})$, and $\operatorname{SAT}(\{U, S, X\},\{\wedge, \vee, \neg\})$ are PSPACEcomplete.

Since there are infinitely many finite sets of Boolean functions, we introduce some algebraic tools to classify the complexity of the infinitely many arising satisfiability problems. We denote with $\mathrm{id}_{k}^{n}$ the $n$-ary projection to the $k$-th variable, i.e., $\operatorname{id}_{k}^{n}\left(x_{1}, \ldots, x_{n}\right)=x_{k}$, and with $c_{a}^{n}$ the $n$-ary constant function defined by $c_{a}^{n}\left(x_{1}, \ldots, x_{n}\right)=a$. For $c_{1}^{1}(x)$ and $c_{0}^{1}(x)$ we simply write 1 and 0 . A set $C$ of Boolean functions is called a clone if it is closed under superposition, which means $C$ contains all projections and $C$ is closed under arbitrary composition [Pip97. For a set $B$ of Boolean functions we denote with $[B]$ the smallest clone containing $B$ and call $B$ a base for $[B]$. In Pos41 Post classified the lattice of all clones Figure 11) and found a finite base for each clone.

We now define some properties of Boolean functions, where $\oplus$ denotes the binary exclusive or.

Definition 2.2. Let $f$ be an n-ary Boolean function.

- $f$ is 1 -reproducing if $f(1, \ldots, 1)=1$.

- $f$ is monotone if $a_{1} \leq b_{1}, \ldots, a_{n} \leq b_{n}$ implies $f\left(a_{1}, \ldots, a_{n}\right) \leq f\left(b_{1}, \ldots, b_{n}\right)$.

- $f$ is 1 -separating if there exists an $i \in\{1, \ldots, n\}$ such that $f\left(a_{1}, \ldots, a_{n}\right)=1$ implies $a_{i}=1$.

- $f$ is self-dual if $f \equiv \operatorname{dual}(f)$, where $\operatorname{dual}(f)\left(x_{1}, \ldots, x_{n}\right)=\neg f\left(\neg x_{1}, \ldots, \neg x_{n}\right)$.

- $f$ is linear if $f \equiv x_{1} \oplus \cdots \oplus x_{n} \oplus c$ for a constant $c \in\{0,1\}$ and variables $x_{1}, \ldots, x_{n}$.

In Table 2 we define those clones that are essential for this paper plus four basic ones, and give Post's bases [Pos41] for them. The inclusions between them are given in Figure 1. The definitions of all clones as well as the full inclusion graph can be found, for example, in [BCRV03].

There is a strong connection between propositional formulae and Post's lattice. If we interpret propositional formulae as Boolean functions, it is obvious that $[B]$ includes 


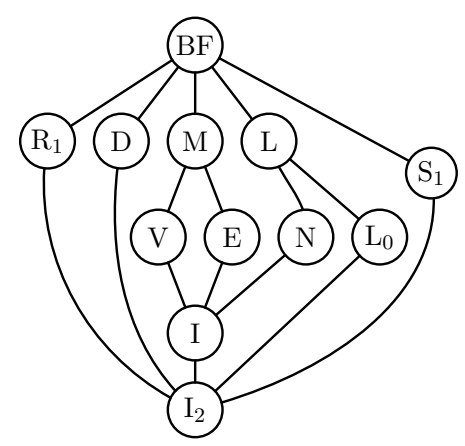

Figure 1: Graph of some closed classes of Boolean functions

\begin{tabular}{lll}
\hline Name & Definition & Base \\
\hline $\mathrm{BF}$ & All Boolean functions & $\{\vee, \wedge, \neg\}$ \\
\hline $\mathrm{R}_{1}$ & $\{f \in \mathrm{BF} \mid f$ is 1-reproducing $\}$ & $\{\vee, \leftrightarrow\}$ \\
\hline $\mathrm{M}$ & $\{f \in \mathrm{BF} \mid f$ is monotone $\}$ & $\{\vee, \wedge, 0,1\}$ \\
\hline $\mathrm{S}_{1}$ & $\{f \in \mathrm{BF} \mid f$ is 1-separating $\}$ & $\{x \wedge \bar{y}\}$ \\
\hline $\mathrm{D}$ & $\{f \mid f$ is self-dual $\}$ & $\{x \bar{y} \vee x \bar{z} \vee(\bar{y} \wedge \bar{z})\}$ \\
\hline $\mathrm{L}$ & $\{f \mid f$ is linear $\}$ & $\{\oplus, 1\}$ \\
\hline $\mathrm{L}_{0}$ & {$[\{\oplus\}]$} & $\{\oplus\}$ \\
\hline $\mathrm{V}$ & $\left\{f \mid\right.$ There is a formula of the form $c_{0} \vee c_{1} x_{1} \vee \cdots \vee c_{n} x_{n}$ & $\{\vee, 1,0\}$ \\
& such that $c_{i}$ are constants for $1 \leq i \leq n$ that describes $\left.f\right\}$ & \\
\hline $\mathrm{E}$ & $\left\{f \mid\right.$ There is a formula of the form $c_{0} \wedge\left(c_{1} \vee x_{1}\right) \wedge \cdots \wedge\left(c_{n} \vee x_{n}\right)$ & $\{\wedge, 1,0\}$ \\
& such that $c_{i}$ are constants for $1 \leq i \leq n$ that describes $\left.f\right\}$ & \\
\hline $\mathrm{N}$ & $\{f \mid f$ depends on at most one variable $\}$ & $\{\neg, 1,0\}$ \\
\hline $\mathrm{I}$ & $\{f \mid f$ is a projection or constant $\}$ & $\{0,1\}$ \\
\hline $\mathrm{I}_{2}$ & $\{f \mid f$ is a projection $\}$ & $\emptyset$ \\
\hline
\end{tabular}

Table 2: List of some closed classes of Boolean functions with bases

exactly those functions that can be represented by $B$-formulae. This connection has been used various times to classify the complexity of problems related to propositional formulae: For example, Lewis presented a dichotomy for the satisfiability problem for propositional $B$-formulae: $\operatorname{SAT}(\emptyset, B)$ is NP-complete if $\mathrm{S}_{1} \subseteq[B]$, and solvable in $\mathrm{P}$ otherwise [Lew79].

Post's lattice was applied for the equivalence problem [Rei01, counting [RW05] and finding minimal [RV03] solutions, and learnability [Dal00] for Boolean formulae. The technique has been used in non-classical logic as well: Bauland et al. achieved a trichotomy in the context of modal logic, which says that the satisfiability problem for modal formulae is, depending on the allowed propositional connectives, PSPACE-complete, coNP-complete, or solvable in $\mathrm{P}$ [BHSS06]. For the inference problem for propositional circumscription, Nordh presented another trichotomy theorem [Nor05.

An important tool in restricting the length of the resulting formula in many of our reductions is the following lemma. It shows that for certain sets $B$, there are always short formulae representing the functions and, or, or not, respectively. Point (2) and (3) follow directly from the proofs in [Lew79, point (1) is Lemma 3.3 from Sch05. 


\section{Lemma 2.3.}

(1) Let $B$ be a finite set of Boolean functions such that $V \subseteq[B] \subseteq \mathrm{M}(E \subseteq[B] \subseteq \mathrm{M}$, resp.). Then there exists a $B$-formula $f(x, y)$ such that $f$ represents $x \vee y$ ( $x \wedge y$, resp.) and each of the variables $x$ and $y$ occurs exactly once in $f(x, y)$.

(2) Let $B$ be a finite set of Boolean functions such that $[B]=\mathrm{BF}$. Then there are $B$ formulae $f(x, y)$ and $g(x, y)$ such that $f$ represents $x \vee y, g$ represents $x \wedge y$, and both variables occur in each of these formulae exactly once.

(3) Let $B$ be a finite set of Boolean functions such that $\mathrm{N} \subseteq[B]$. Then there is a B-formula $f(x)$ such that $f$ represents $\neg x$ and the variable $x$ occurs in $f$ only once.

\section{Results}

Our proofs for most of the upper complexity bounds will rely on similar ideas as the ones in [BHSS06, which are extensions of the proof techniques for the polynomial time results in [Lew79]. However, the proof of our polynomial time result for formulae using the exclusive or (Theorem 3.8) will be unrelated to the positive cases for XOR in the mentioned papers.

The proofs for hardness results will use different techniques. Hardness proofs for unimodal logics usually work in embedding a tree-like structure directly into a tree-like model for modal formulae. Naturally, this approach does not work with LTL which speaks about linear models. Hence, in the proof of Theorem 3.3, we will encode a tree-like structure into a linear one, and most of the complexity of the proof will come from the need to enforce a tree-like behavior of linear models.

3.1. Hard cases. The following lemma gives our general upper bounds for various combinations of temporal operators. It establishes that the known upper complexity bounds for the case where only the propositional operators and, or, and negation are allowed to appear in the formulae still hold for the more general cases that we consider. This does not follow trivially, since there is no obvious strategy that converts every $B$-formula into a formula using only the standard connectives without leading to an exponential increase in formula length. The issues here are similar to the "succinctness gap" between the logics LTL+Past and LTL discussed in Mar04. The proof of Parts (1) and (2) of the following lemma is a variation of the proof for Theorem 3.4 in [BHSS06], where, using a similar reduction, an analogous result for circuits was proved.

Lemma 3.1. Let $B$ be a finite set of Boolean functions. Then the following holds:

(1) If $M \subseteq\{\mathrm{F}, \mathrm{G}, \mathrm{U}, \mathrm{S}, \mathrm{X}\}$, then $\operatorname{SAT}(M, B)$ is in PSPACE,

(2) if $M \subseteq\{\mathrm{F}, \mathrm{G}\}$, then $\operatorname{SAT}(M, B)$ is in $\mathrm{NP}$, and

(3) if $M \subseteq\{\mathrm{X}\}$, then $\operatorname{SAT}(M, B)$ is also in NP.

Proof. For (1), we will show that $\operatorname{SAT}(M, B) \leq_{m}^{\log } \operatorname{SAT}(\{\mathrm{U}, \mathrm{S}, \mathrm{X}\},\{\wedge, \vee, \neg\})$, and for (2), we will show that $\operatorname{SAT}(M, B) \leq_{m}^{\log } \operatorname{SAT}(\{\mathrm{F}\},\{\wedge, \vee, \neg\})$. The complexity result for these cases then follows from Theorem 2.1.

The construction for (1) and (2) is nearly identical: Let $\varphi$ be a formula with arbitrary temporal operators and Boolean functions from $B$. We recursively transform the formula to a new formula using only the Boolean operators $\wedge, \vee$, and $\neg$, and the temporal operators $\mathrm{U}, \mathrm{S}$, and $\mathrm{X}$ for the first case and the temporal operator $\mathrm{F}$ for the second cases. For this we 
construct several formulae, which will be connected via conjunction. Let $k$ be the number of subformulae of $\varphi$. Accordingly let $\varphi_{1}, \ldots, \varphi_{k}$ be those subformulae with $\varphi=\varphi_{1}$. Let $x_{1}, \ldots, x_{k}$ be new variables, i.e., distinct from the input variables of $\varphi$. For all $i$ from 1 to $k$ we make the following case distinction:

- If $\varphi_{i}=y$ for a variable $y$, then let $f_{i}(\varphi)=x_{i} \leftrightarrow y$.

- If $\varphi_{i}=\mathrm{X} \varphi_{j}$, then let $f_{i}(\varphi)=x_{i} \leftrightarrow \mathrm{X} x_{j}$.

- If $\varphi_{i}=\mathrm{F} \varphi_{j}$, then let $f_{i}(\varphi)=x_{i} \leftrightarrow \mathrm{F} x_{j}$.

- If $\varphi_{i}=\mathrm{G} \varphi_{j}$, then let $f_{i}(\varphi)=x_{i} \leftrightarrow \mathrm{G} x_{j}$.

- If $\varphi_{i}=\varphi_{j} \mathrm{U} \varphi_{\ell}$, then let $f_{i}(\varphi)=x_{i} \leftrightarrow x_{j} \mathrm{U} x_{\ell}$.

- If $\varphi_{i}=\varphi_{j} \mathrm{~S} \varphi_{\ell}$, then let $f_{i}(\varphi)=x_{i} \leftrightarrow x_{j} \mathrm{~S} x_{\ell}$.

- If $\varphi_{i}=g\left(\varphi_{i_{1}}, \ldots, \varphi_{i_{n}}\right)$ for some $g \in B$, then let $f_{i}(\varphi)=x_{i} \leftrightarrow h\left(x_{i_{1}}, \ldots, x_{i_{n}}\right)$, where $h$ is a formula using only $\wedge, \vee$, and $\neg$, representing the function $g$.

Such a formula $h$ always exists with constant length, because the set $B$ is fixed and does not depend on the input. Now let $f(\varphi)=x_{1} \wedge \bigwedge_{i=1}^{k}\left(\mathrm{G} f_{i}(\varphi) \wedge \neg\left(\operatorname{true} \mathrm{S} \neg f_{i}(\varphi)\right)\right)$ for case (1) and $f(\varphi)=x_{1} \wedge \bigwedge_{i=1}^{k} \mathrm{G} f_{i}(\varphi)$ for case (2). The part $\mathrm{G} f_{i}(\varphi)$ makes sure that $f_{i}(\varphi)$ holds in every future state of the structure and $\left.\neg\left(\operatorname{true} S \neg f_{i}(\varphi)\right)\right)$ does the same for the past states of the structure. Additionally we consider $x \leftrightarrow y$ as a shorthand for $(x \wedge y) \vee(\neg x \wedge \neg y)$. For case (1) we consider $\mathrm{F} x$ as a shorthand for true $\mathrm{U} x$ and $\mathrm{G} x$ as a shorthand for $\neg(\operatorname{true} \mathrm{U} \neg x)$, and for case (2) we consider $\mathrm{G} x$ as a shorthand for $\neg \mathrm{F} \neg x$. Thus we have that $f(\varphi)$ is from $\mathrm{L}(\{\mathrm{U}, \mathrm{S}, \mathrm{X}\},\{\wedge, \vee, \neg\})$ in case $(1)$ and from $\mathrm{L}(\{\mathrm{F}\},\{\wedge, \vee, \neg\})$ in case (2). Furthermore $f$ is computable in logarithmic space, because the length of $f_{i}$ is polynomial and neither $\leftrightarrow$ nor the formulae $h$ occur nested. In order to show that $f$ is the reduction we are looking for, we still need to prove that $\varphi$ is satisfiable if and only if $f(\varphi)$ is satisfiable. Assume an arbitrary structure $S$, such that $S, s_{i} \vDash f(\varphi)$ for some $s_{i}$. We first prove by induction on the structure of the formula that $x_{i}$ holds if and only if $\varphi_{i}$ holds in every state $s$ of $S$ (for (1)) respectively in every state which lies in the future of $s_{i}$ (for (2)). Therefore for (1) let $s$ be an arbitrary state and for (2) let $s$ be an arbitrary state in the future of $s_{i}$. Thus by construction of $f(\varphi)$ the formulae $f_{p}(\varphi)$ hold at $s$ for all $1 \leq p \leq k$. Then the following holds:

- If $\varphi_{p}=y$ for a variable $y$, then $f_{p}(\varphi)=x_{p} \leftrightarrow y$ and trivially $S, s \vDash x_{p}$ iff $S, s \vDash y$.

- If $\varphi_{p}=\mathrm{X} \varphi_{j}$, then $f_{p}(\varphi)=x_{p} \leftrightarrow \mathbf{X} x_{j}$. Thus $S, s \vDash x_{p}$ iff for the successor state $s^{\prime}$ of $s$, we have $S, s^{\prime} \vDash x_{j}$. By induction this is equivalent to $S, s^{\prime} \vDash \varphi_{j}$ and therefore $S, s \vDash \varphi_{p}$ iff $S, s \vDash x_{p}$.

- The cases for the temporal operator $\mathrm{F}$ or $\mathrm{G}$ work analogously.

- If $\varphi_{p}=\varphi_{j} \cup \varphi_{\ell}$, then $f_{p}(\varphi)=x_{p} \leftrightarrow x_{j} \cup x_{\ell}$. Thus $S, s \vDash x_{p}$ iff there exists a state $s^{\prime}$ in the future of $s$, such that $S, s^{\prime} \vDash x_{\ell}$ and in all states $s_{m}$ in between (including $s$ ) $S, s_{m} \vDash x_{j}$. By induction this is equivalent to $S, s^{\prime} \vDash \varphi_{\ell}$ and for all states in between $S, s_{m} \vDash \varphi_{j}$ and therefore $S, s \vDash \varphi_{p}$ iff $S, s \vDash x_{p}$.

- If $\varphi_{p}=\varphi_{j} \mathrm{~S} \varphi_{\ell}$, then $f_{p}(\varphi)=x_{p} \leftrightarrow x_{j} \mathrm{~S} x_{\ell}$. Thus $S, s \vDash x_{p}$ iff there exists a state $s^{\prime}$ in the past of $s$, such that $S, s^{\prime} \vDash x_{\ell}$ and in all states $s_{m}$ in between (including $\left.s\right) S, s_{m} \vDash x_{j}$. By induction this is equivalent to $S, s^{\prime} \vDash \varphi_{\ell}$ and for all states in between $S, s_{m} \vDash \varphi_{j}$ and therefore $S, s \vDash \varphi_{p}$ iff $S, s \vDash x_{p}$.

- If $\varphi_{p}=g\left(\varphi_{i_{1}}, \ldots, \varphi_{i_{n}}\right)$, then $f_{p}(\varphi)=x_{p} \leftrightarrow h\left(x_{i_{1}}, \ldots, x_{i_{n}}\right)$, where $h$ is a formula using only $\wedge, \vee$, and $\neg$, representing the function $g$. Thus $S, s \vDash x_{p}$ iff $S, s \vDash h\left(x_{i_{1}}, \ldots, x_{i_{n}}\right)$. Let $I$ be the subset of $I^{n}=\left\{i_{1}, \ldots, i_{n}\right\}$, such that $S, s \vDash x_{m}$ for all $m \in I$ and $S, s \vDash \neg x_{m}$ for all $m \in I^{n} \backslash I$. By induction $S, s \vDash \varphi_{m}$ for all $m \in I$ and $S, s \vDash \neg \varphi_{m}$ for all $m \in I^{n} \backslash I$ and 
therefore $S, s \vDash h\left(\varphi_{i_{1}}, \ldots, \varphi_{i_{n}}\right)$. Since $h$ represents the function $g$, we have that $S, s \vDash \varphi_{p}$ iff $S, s \vDash x_{p}$.

Now, assume that $f(\varphi)$ is satisfiable. Then there exists a structure $S, s_{i} \vDash f(\varphi)$ and thus $S, s_{i} \vDash x_{1}$. Since in every state $x_{j}$ holds if and only if $\varphi_{j}$ holds, we have that $S, s_{i} \vDash$ $\varphi=\varphi_{1}$. For the other direction, assume that $\varphi$ is satisfiable. Then there exists a structure $S, s_{i} \vDash \varphi=\varphi_{1}$. Now we can extend $S$ by adding new variables $x_{1}, \ldots, x_{k}$ in such a way, that $x_{j}$ holds in a state $s$ from $S$ if and only if $\varphi_{j}$ holds in that state. Call this new structure $S^{\prime}$. Then by construction of $f(\varphi)$, we have $S^{\prime}, s_{i} \vDash f(\varphi)$, since in every state $x_{j}$ holds if and only if $\varphi_{j}$ holds. This concludes the proof of the first two cases.

We now show (3). For a formula $\varphi$ in which $X$ is the only temporal operator, let $\operatorname{depth}_{X}(\varphi)$ denote the maximal nesting degree of the $\mathbf{X}$-operator in $\varphi$, which we call the $\mathbf{X}$-depth of $\varphi$. It is obvious that this number is linear in the length of $\varphi$. Therefore, to show that the problem can be solved in NP, it suffices to prove the following:

(a) Such a formula $\varphi$ is satisfiable if and only if there is a structure $S$ with the sequence $\left(s_{i}\right)_{i \in \mathbb{N}}$ such that for every $i>\operatorname{depth}_{\mathbf{X}}(\varphi)$, every variable in $s_{i}$ is false, and $S, s_{0} \models \varphi$.

(b) Given the assignments to the variables in the first $\operatorname{depth}_{X}(\varphi)$ states in the structure above, it can be verified in polynomial time if $S, s_{0} \models \varphi$.

These claims immediately imply the complexity result. For the first point, it obviously suffices to show one direction. Therefore, let $S$ be an arbitrary structure with sequence $\left(s_{i}\right)_{i \in \mathbb{N}}$ such that $S, s_{0} \models \varphi$, and let $S^{\prime}$ be the structure with sequence $\left(s_{i}^{\prime}\right)_{i \in \mathbb{N}}$ obtained from $S$ as follows: For $i \leq \operatorname{depth}_{\mathrm{X}}(\varphi)$, the assignment of the variables in the state $s_{i}^{\prime}$ is the same as in $s_{i}$. For $i>\operatorname{depth}_{\mathbf{X}}(\varphi)$, every variable is false in $s_{i}^{\prime}$. To prove claim (a) above, it suffices to prove that $S^{\prime}, s_{0}^{\prime} \models \varphi$.

To show this, we prove that for every subformula $\psi$ of $\varphi$ and every $i \leq \operatorname{depth}_{\mathbf{X}}(\varphi)$, if $\operatorname{depth}_{\mathbf{X}}(\psi) \leq \operatorname{depth}_{\mathbf{X}}(\varphi)-i$, then $S, s_{i} \models \psi$ if and only if $S^{\prime}, s_{i}^{\prime} \models \psi$. For $i=0$ and $\psi=\varphi$, this implies the desired result $S^{\prime}, s_{0}^{\prime} \models \varphi$.

We show the claim by induction on the formula $\psi$. If $\psi$ is a variable, then, by construction, $S^{\prime}, s_{i}^{\prime} \models \psi$ if and only if $S, s_{i} \models \psi$, since the truth assignments of $s_{i}^{\prime}$ and $s_{i}$ are identical. Now let $\psi$ be of the form $f\left(\psi_{1}, \ldots, \psi_{n}\right)$ for an $n$-ary function $f \in B$. In this case, it immediately follows that $\operatorname{depth}_{\mathbf{X}}(\psi)=\max \left\{\operatorname{depth}_{\mathbf{X}}\left(\psi_{1}\right), \ldots, \operatorname{depth}_{\mathbf{X}}\left(\psi_{n}\right)\right\}$. Because of the prerequisites, $\operatorname{depth}_{\mathbf{X}}(\psi) \leq \operatorname{depth}_{\mathbf{X}}(\varphi)-i$, and hence we know that for each $j \in\{1, \ldots, n\}$, it holds that $\operatorname{depth}_{\mathbf{X}}\left(\psi_{j}\right) \leq \operatorname{depth}_{\mathbf{X}}(\varphi)-i$. Therefore, we can apply the induction hypothesis to all of the $\psi_{j}$, and we know that $S, s_{i} \models \psi_{j}$ if and only if $S^{\prime}, s_{i}^{\prime} \models \psi_{j}$. This immediately implies that $S, s_{i} \models \psi$ if and only if $S^{\prime}, s_{i}^{\prime} \models \psi$, since $f$ is a Boolean function.

Finally, let $\psi$ be of the form $\mathbf{X} \xi$ for some formula $\xi$. Hence, $\operatorname{depth}_{\mathbf{X}}(\psi)=\operatorname{depth}_{\mathbf{X}}(\xi)+1$. Since $\operatorname{depth}_{\mathbf{X}}(\psi) \leq \operatorname{depth}_{\mathbf{X}}(\varphi)-i$, this $\operatorname{implies}$ that $\operatorname{depth}_{\mathbf{X}}(\xi) \leq \operatorname{depth}_{\mathbf{X}}(\varphi)-(i+1)$. Hence, we can apply the induction hypothesis, and conclude that $S, s_{i+1} \models \xi$ if and only if $S^{\prime}, s_{i+1}^{\prime} \models \xi$. This immediately implies that $S, s_{i} \models \psi$ if and only if $S^{\prime}, s_{i}^{\prime} \models \psi$, and hence concludes the induction and the proof of claim (a).

For claim (b), assume that $\varphi$ and the truth assignments for the first $\operatorname{depth}_{\mathbf{X}}(\varphi) \operatorname{states}$ in the structure $S$ are given, where all variables are assumed to be false in all further states. We can now, for each subformula $\psi$ of $\varphi$, mark those states $s_{i}\left(\right.$ for $i \leq \operatorname{depth}_{\mathbf{X}}(\varphi)$ ) in which $\psi$ holds. Starting with $j=0$, consider the subformulae of $\mathbf{X}$-depth $j$. The question if a formula of $\mathbf{X}$-depth $j$ holds at a given state can easily be decided when this is known for all formulae of lower $\mathbf{X}$-depth. For $j=0$, this can be decided easily, since the subformulae of $\mathrm{X}$-depth 0 are exactly the propositional subformulae, and for these, each state can be 
considered separately. Additionally, observe that in the structure $S$, all states beyond the first $\operatorname{depth}_{X}(\varphi)$ states satisfy exactly the same set of subformulae of $\varphi$, hence only $\operatorname{depth}_{\mathbf{X}}(\varphi)+1$ many states need to be considered.

The following two theorems show that the case in which our Boolean operators are able to express the function $x \wedge \bar{y}$, leads to PSPACE-complete problems in the same cases as for the full set of Boolean operators. This function already played an important role in the classification result from [Lew79], where it also marked the "jump" in complexity from polynomial time to NP-complete.

Theorem 3.2. Let $B$ be a finite set of Boolean functions such that $\mathrm{S}_{1} \subseteq[B]$. Then $\mathrm{SAT}(\{\mathrm{G}, \mathrm{X}\}, B)$ and $\mathrm{SAT}(\{\mathrm{F}, \mathrm{X}\}, B)$ are PSPACE-complete.

Proof. Since it is possible to express $F$ using $G$ and negation, Theorem 2.1 implies that $\operatorname{SAT}(\{G, X\},\{\wedge, \vee, \neg\})$ and $\operatorname{SAT}(\{F, X\},\{\wedge, \vee, \neg\})$ are PSPACE-hard. Now, let $\varphi$ be a formula in which only temporal operators $G$ and $X$, or $F$ and $X$, and the Boolean connectives $\wedge, \vee$, and $\neg$ appear. Let $B^{\prime}=B \cup\{1\}$. The complete structure of Post's lattice [BCRV03] shows that $\left[B^{\prime}\right]=B F$. Now we can rewrite $\varphi$ as a $B^{\prime}$-formula with the same temporal operators appearing. Due to Lemma 2.3, we can express the crucial operators $\wedge, \vee, \neg$ with short $B^{\prime}$-formulae, i.e., formulae in which every relevant variable occurs only once. Therefore, this transformation can be performed in polynomial time. Now, in the $B^{\prime}$-representation of $\varphi$, we exchange every occurrence of 1 with a new variable $t$, and call the result $\varphi^{\prime}$, which is a $B$-formula. It is obvious that $\varphi$ is satisfiable if and only if the $B$-formula $\varphi^{\prime} \wedge t \wedge \mathrm{G} t$ is. Since $B \supseteq \mathrm{S}_{1}$, we can express the occurring conjunctions using operators from $B$ (since these are a constant number of conjunctions, we do not need to worry about needing long $B$-formulae to express conjunction). This finishes the proof for $\operatorname{SAT}(\{\mathrm{G}, \mathrm{X}\}, B)$. For the problem $\operatorname{SAT}(\{\mathrm{F}, \mathrm{X}\}, B)$, observe that the function $g(x, y)=x \wedge \bar{y}$ generates the clone $\mathrm{S}_{1}$, and therefore there is some $B$-formula equivalent to $g$. Now observe that the formula $t \wedge \overline{\mathrm{F}(t \wedge \overline{\mathrm{X} t})}=g(t, \mathrm{~F}(g(t, \mathrm{X} t)))$ is equivalent to $\mathrm{G} t$. Since this formula is independent of the input formula $\varphi$, this can be computed in polynomial time, and therefore this formula can be used to express $\varphi^{\prime} \wedge t \wedge \mathrm{G} t$ in the same way as in the first case. Additionally, observe that if the operator $\mathrm{F}$ appears in the original formula $\varphi$, then a subformula $\mathrm{F} \psi$ can be expressed as $(1 \mathrm{U} \psi)$. Hence we conclude from Theorem 2.12 that $\operatorname{SAT}(\{\mathrm{U}, \mathrm{X}\}, \mathrm{BF})$ is PSPACE-complete.

The construction in the proof of Theorem 3.2 does not seem to be applicable to the languages with $\mathrm{U}$ and/or $\mathrm{S}$, as it requires a way to express $\mathrm{G} t$ using these operators. Hence, proving the desired completeness result requires significantly more work. Note that the case where $B$ contains the usual operators and, or, and negation, has already been proved in Mar04. Our construction shows that hardness already holds for a class of propositional operators with less expressive power.

Theorem 3.3. Let $B$ be a finite set of Boolean functions with $\mathrm{S}_{1} \subseteq[B]$. Then $\operatorname{SAT}(\{\mathrm{S}\}, B)$ and $\operatorname{SAT}(\{\mathrm{U}\}, B)$ are PSPACE-complete.

Proof. Since membership for PSPACE is shown in Lemma 3.1 we only need to show hardness. To do this, we give a reduction from QBF. The main idea is to construct a temporal $B$ formula that requires satisfying models to simulate, in a linear structure, the quantifier evaluation tree of a quantified Boolean formula. Once we have ensured that models for the formula in fact are of this structure, we can prove that the quantified formula evaluation problem reduces to $\mathrm{SAT}(\{\mathrm{S}\}, B)$. 
First we prove an auxiliary proposition for formulae of a special form which we use as building blocks in the construction. Intuitively the claim states that, given some propositional formulae $\varphi_{1}, \ldots, \varphi_{n}$ that are pairwise contradictory, we can express that a model has a subsequence of states such that $\varphi_{i}$ holds in the $i$-th of these states.

We cannot enforce that the $i$-th state always satisfies the $i$-th formula, since the truth of an LTL-formula using only $S$ as a temporal operator is invariant under transformations of models that simply repeat a state finitely many times in the sequence.

Claim 1. Let $\varphi_{1}, \ldots, \varphi_{n}$ be satisfiable propositional formulae such that $\varphi_{i} \rightarrow \neg \varphi_{j}$ is valid for all $i, j \in\{1, \ldots, n\}$ with $i \neq j$. Then the formula

$$
\varphi=\varphi_{1} \wedge\left(\varphi_{1} \mathrm{~S}\left(\varphi_{2} \mathrm{~S}\left(\ldots \mathrm{S}\left(\varphi_{n-1} \mathrm{~S} \varphi_{n}\right) \ldots\right)\right) \wedge\left(\left(\ldots\left(\left(\varphi_{1} \mathrm{~S} \varphi_{2}\right) \mathrm{S} \varphi_{3}\right) \mathrm{S} \ldots\right) \mathrm{S} \varphi_{n}\right)\right.
$$

is satisfiable and every structure $S$ that satisfies $\varphi$ in a state $s_{m}$ fulfills the following property: there exist natural numbers $0=a_{0}<a_{1}<\cdots<a_{n} \leq m+1$ such that $m-a_{i}<j \leq m-a_{i-1}$ implies $S, s_{j} \vDash \varphi_{i}$ for every $i \in\{1 \ldots, n\}$.

Proof. Clearly $\varphi$ is satisfiable: since all formulae $\varphi_{i}$ are satisfiable we can find a structure $S$ such that $S, s_{i} \vDash \varphi_{n-i}$ for all $i \in\{0, \ldots, n-1\}$. One can verify that $S$ satisfies $\varphi$ in $s_{n-1}$.

Let $S$ be a structure that satisfies $\varphi$ in a state $s_{m}$. Since $\varphi_{i} \rightarrow \neg \varphi_{j}$ is valid for all $i, j \in\{1, \ldots, n\}$ with $i \neq j$, in every state only one of the formulae $\varphi_{i}$ can be satisfied by $S$. Therefore and since $S, s_{m} \vDash \varphi_{1} \mathrm{~S}\left(\varphi_{2} \mathrm{~S}\left(\ldots \mathrm{S}\left(\varphi_{n-1} \mathrm{~S} \varphi_{n}\right) \ldots\right)\right)$ holds, there are natural numbers $0=a_{0} \leq a_{1} \leq \cdots \leq a_{n-1}<a_{n} \leq m+1$ such that $m-a_{i}<l \leq m-a_{i-1}$ implies $S, s_{l} \vDash \varphi_{i}$ for every $i \in\{1 \ldots, n\}$. Since $S, s_{m} \vDash \varphi_{1}$, it holds that $a_{1}>0$. Because $S, s_{m} \vDash\left(\ldots\left(\left(\varphi_{1} S \varphi_{2}\right) \mathrm{S} \varphi_{3}\right) \mathrm{S} \ldots\right) \mathrm{S} \varphi_{n}$ we conclude that $a_{1}<\cdots<a_{n-1}$, which proves the claim.

Now we give the reduction from QBF, which is PSPACE-complete due to [Sto77, to $\operatorname{SAT}(\{\mathrm{S}\}, B)$. Let $\psi=Q_{1} x_{1} \ldots Q_{n} x_{n} \varphi$ for some propositional $\{\wedge, \vee, \neg\}$-formula $\varphi$ with variables $x_{1}, \ldots, x_{n}$ and for quantifiers $Q_{1}, \ldots, Q_{n} \in\{\forall, \exists\}$.

Let $I_{\forall}=\left\{p_{1}, \ldots, p_{k}\right\}=\left\{i \mid Q_{i}=\forall\right\}$ and $I_{\exists}=\left\{q_{1}, \ldots, q_{l}\right\}=\left\{i \mid Q_{i}=\exists\right\}$ such that $p_{1}<\cdots<p_{k}$ and $q_{1}<\cdots<q_{l}$.

We construct a temporal formula $\psi^{\prime} \in \mathrm{L}(\{\mathrm{S}\}, B)$ such that $\psi$ is valid if and only if $\psi^{\prime}$ is satisfiable. Let $t_{0}, \ldots, t_{n}, u_{0}, \ldots, u_{n}$ be new variables. We start with defining some subformulae using propositional operators from $\{\neg, \vee, \wedge\}$, then we combine them to obtain $\psi^{\prime}$, and afterwards turn $\psi^{\prime}$ into a temporal $B$-formula.

$$
\begin{aligned}
& \left.\alpha=u_{0} \wedge \overline{t_{0}} \wedge\left(u_{0} \wedge \overline{t_{0}}\right) \mathrm{S}\left(\left(\overline{u_{0}} \wedge \overline{t_{0}}\right) \mathrm{S}\left(\overline{u_{0}} \wedge t_{0}\right)\right)\right) \wedge\left(\left(\left(u_{0} \wedge \overline{t_{0}}\right) \mathrm{S}\left(\overline{u_{0}} \wedge \overline{t_{0}}\right)\right) \mathrm{S}\left(\overline{u_{0}} \wedge t_{0}\right)\right) \\
& \beta^{1}[i]= \\
& \left(u_{i-1} \wedge \overline{t_{i-1}} \wedge u_{i} \wedge \overline{t_{i}} \wedge \overline{x_{i}}\right) \mathrm{S} \\
& \left(\left(\overline{u_{i-1}} \wedge \overline{t_{i-1}} \wedge \overline{u_{i}} \wedge \overline{t_{i}} \wedge \overline{x_{i}}\right) \mathrm{S}\right. \\
& \left(\left(\overline{u_{i-1}} \wedge \overline{t_{i-1}} \wedge \overline{u_{i}} \wedge t_{i} \wedge \overline{x_{i}}\right) \mathrm{S}\right. \\
& \left(\left(\overline{u_{i-1}} \wedge \overline{t_{i-1}} \wedge u_{i} \wedge \overline{t_{i}} \wedge x_{i}\right) \mathrm{S}\right. \\
& \left(\left(\overline{u_{i-1}} \wedge \overline{t_{i-1}} \wedge \overline{u_{i}} \wedge \overline{t_{i}} \wedge x_{i}\right) \mathrm{S}\right. \\
& \beta^{2}[i]= \\
& \left.\left.\left.\left.\left(\overline{u_{i-1}} \wedge t_{i-1} \wedge \overline{u_{i}} \wedge t_{i} \wedge x_{i}\right)\right)\right)\right)\right) \\
& \left(\left(\left(\left(u_{i-1} \wedge \overline{t_{i-1}} \wedge u_{i} \wedge \overline{t_{i}} \wedge \overline{x_{i}}\right)\right.\right.\right. \\
& \left.\mathrm{S}\left(\overline{u_{i-1}} \wedge \overline{t_{i-1}} \wedge \overline{u_{i}} \wedge \overline{t_{i}} \wedge \overline{x_{i}}\right)\right) \\
& \left.\mathrm{S}\left(\overline{u_{i-1}} \wedge \overline{t_{i-1}} \wedge \overline{u_{i}} \wedge t_{i} \wedge \overline{x_{i}}\right)\right) \\
& \left.\mathrm{S}\left(\overline{u_{i-1}} \wedge \overline{t_{i-1}} \wedge u_{i} \wedge \overline{t_{i}} \wedge x_{i}\right)\right) \\
& \left.\mathrm{S}\left(\overline{u_{i-1}} \wedge \overline{t_{i-1}} \wedge \overline{u_{i}} \wedge \overline{t_{i}} \wedge x_{i}\right)\right) \\
& \mathrm{S}\left(\overline{u_{i-1}} \wedge t_{i-1} \wedge \overline{u_{i}} \wedge t_{i} \wedge x_{i}\right)
\end{aligned}
$$




$$
\begin{aligned}
& \gamma^{1}[i]=\left(u_{i-1}\right.\left.\wedge \overline{t_{i-1}} \wedge u_{i} \wedge \overline{t_{i}} \wedge \overline{x_{i}}\right) \mathrm{S} \\
&\left(\left(\overline{u_{i-1}} \wedge \overline{t_{i-1}} \wedge \overline{u_{i}} \wedge \overline{t_{i}} \wedge \overline{x_{i}}\right) \mathrm{S}\right. \\
&\left.\left(\left(\overline{u_{i-1}} \wedge t_{i-1} \wedge \overline{u_{i}} \wedge t_{i} \wedge \overline{x_{i}}\right)\right)\right)
\end{aligned}
$$

$$
\begin{aligned}
& \gamma^{2}[i]=\left(u_{i-1}\right.\left.\wedge \overline{t_{i-1}} \wedge u_{i} \wedge \overline{t_{i}} \wedge x_{i}\right) \mathrm{S} \\
&\left(\left(\overline{u_{i-1}} \wedge \overline{t_{i-1}} \wedge \overline{u_{i}} \wedge \overline{t_{i}} \wedge x_{i}\right) \mathrm{S}\right. \\
&\left.\left(\left(\overline{u_{i-1}} \wedge t_{i-1} \wedge \overline{u_{i}} \wedge t_{i} \wedge x_{i}\right)\right)\right)
\end{aligned}
$$

The formula $\alpha$ initializes a model as follows: it sets $u_{0} \overline{t_{0}}$ in the current state and requires that in the past there is a state with $\overline{u_{0}} t_{0}$ and all states in between satisfy $\overline{u_{0}} \overline{t_{0}}$. We will use $\beta^{1}[i]$ and $\beta^{2}[i]$ for $\forall$-quantified variables $x_{i}$ to partition the states such that $x_{i}$ is true in one partition and false in the other. Finally, we need $\gamma^{1}[i]$ and $\gamma^{2}[i]$ to set the values for the $\exists$-quantified variables.

We now define the formula $\psi^{\prime}$, which constitutes the reduction.

$$
\psi^{\prime}=\alpha \wedge \bigwedge_{i \in I_{\forall}}\left(\left(\beta^{1}[i] \wedge \beta^{2}[i]\right) \mathrm{S} t_{0}\right) \wedge \bigwedge_{i \in I_{\exists}}\left(\left(\gamma^{1}[i] \vee \gamma^{2}[i]\right) \mathrm{S} t_{0}\right) \wedge\left(\varphi \mathrm{S} t_{0}\right)
$$

The formula $\psi^{\prime}$ as defined above is specified as a formula using the connectives and, or, and not. Before proving the correctness of the reduction, we show how $\psi^{\prime}$ can be rewritten using only the available connectives from $B$. Due to the prerequisites, we know that $\mathrm{S}_{1} \subseteq[B]$. From the complete structure of Post's lattice [BCRV03, it follows that $[B \cup\{1\}]=\mathrm{BF}$. Let $B^{\prime}$ denote the set $B \cup\{1\}$. Since, due to Lemma 2.3, conjunction, disjunction, and negation can be written as $B^{\prime}$-formulae such that every relevant variable appears only once, we can rewrite $\psi^{\prime}$ into a temporal $B^{\prime}$-formula with the result growing only polynomially in size (and the transformation can be carried out in polynomial time). Hence we can regard $\psi^{\prime}$ as a temporal $B^{\prime}$-formula. Now, since $[B] \supseteq \mathrm{S}_{1}$, and the and-function is an element of $\mathrm{S}_{1}$, there is a $B$-formula and ${ }_{B}(x, y)$ which is equivalent to $x \wedge y$ (but both $x$ and $y$ might occur more than once in $\left.\operatorname{and}_{B}(x, y)\right)$. Now consider the propositional conjunctions of up to 5 literals occurring in the subformulae $\beta^{j}[i], \gamma^{j}[i]$, and $\alpha$ of $\psi^{\prime}$, and recall that in the above step, we have rewritten these into formulae that only use connectives from $B$ and the constant 1 . For each such conjunction $\psi_{\text {lit }}$, let $\psi_{\text {lit }}^{t}$ be the formula obtained from $\psi_{\text {lit }}$ by exchanging each occurrence of the constant 1 with the new variable $t$. Now the formula $\operatorname{and}_{B}\left(t, \psi_{\text {lit }}^{t}\right)$ is equivalent to $\psi_{\text {lit }} \wedge t$. We can therefore replace all formulae $\psi_{\text {lit }}$ with $\operatorname{and}_{B}\left(t, \psi_{\text {lit }}^{t}\right)$, and obtain a formula which is equivalent to $\psi^{\prime}$, but additionally forces the new variable $t$ to true in all the affected states. The remaining conjunctions occurring in the subformula $\alpha$ can simply be rewritten using the $\operatorname{and}_{B}(x, y)$-formula - there is only a constant number of these, hence this rewriting can be done in polynomial time.

It remains to deal with conjunctions on the outmost level of $\psi^{\prime}$, i.e., the three conjunctions connecting the different parts of the formula and the conjunctions over all $i \in I_{\forall}$ and $i \in I_{\exists}$. We first re-arrange these conjunctions as a formula which is a binary tree of logarithmic depth. Then each conjunction can be replaced by using the formula $\operatorname{and}_{B}(x, y)$ defined above. Since the nesting degree of the conjunction (and hence of applications of $\left.\operatorname{and}_{B}(x, y)\right)$ is only logarithmic, this transformation leads to a formula which is polynomial in the length of the original representation of $\psi^{\prime}$, and can be carried out in polynomial time.

The result of these transformations is a temporal $B$-formula which is equivalent to $\psi^{\prime}$, apart from forcing the newly-introduced variable $t$ to true in all worlds in all models of $\psi^{\prime}$ that lie in the scope of the relevant temporal operators. In particular, this formula is satisfiability-equivalent to $\psi^{\prime}$. Hence it suffices to prove that the reduction is correct with respect to $\psi^{\prime}$, i.e., that $\psi^{\prime}$ is satisfiable if and only if the original QBF-instance $\psi$ evaluates 
to true. For this, we first give a characterization of models satisfying $\psi^{\prime}$, which establishes that models for this formula are indeed "flat versions of quantifier-trees."

Hence assume that $S$ is a structure that satisfies $\psi^{\prime}$ in a state $s_{m}$. We prove by induction over $n$ that there are natural numbers $0=a_{0}<\cdots<a_{3\left(2^{k}\right)} \leq m+1$ and for every $q \in I_{\exists}$ a function $\sigma_{q}:\{0,1\}^{q-1} \rightarrow\{0,1\}$ such that $S$ satisfies the following property: if $m-a_{i}<j \leq m-a_{i-1}$, then it holds for all $h$ that

(1) $S, s_{j} \vDash x_{p_{h}}$ iff $\left\lceil\frac{i}{3\left(2^{k-h}\right)}\right\rceil$ is even,

(2) $S, s_{j} \vDash x_{q_{h}}$ iff $\sigma_{q_{h}}\left(a_{1} \ldots, a_{q_{h}-1}\right)=1$ where $a_{d}=1$ if $x_{d} \in \xi\left(s_{j}\right)$ and $a_{d}=0$ otherwise,

(3) $S, s_{j} \vDash t_{0}$ iff $i=3\left(2^{k}\right)$,

(4) $S, s_{j} \vDash t_{p_{h}}$ iff $i=c \cdot 3\left(2^{k-h}\right)$ for some $c \in \mathbb{N}$,

(5) $S, s_{j} \vDash t_{q_{h}}$ iff $S, s_{j} \vDash t_{p_{h}-1}$,

(6) $S, s_{j} \vDash u_{0}$ iff $i=1$,

(7) $S, s_{j} \vDash u_{p_{h}}$ iff $i=c \cdot 3\left(2^{k-h}\right)+1$ for some $c \in \mathbb{N}$,

(8) $S, s_{j} \vDash u_{q_{h}}$ iff $S, s_{j} \vDash u_{p_{h}-1}$.

Note that due to point 1 for every possible assignment $\pi$ to $\left\{x_{p_{1}}, \ldots, x_{p_{k}}\right\}$ there is a $j \in$ $\left\{m-a_{3\left(2^{k}\right)}+1, \ldots, m\right\}$ such that $S, s_{j} \vDash x_{p_{i}}$ if and only if $\pi\left(x_{p_{i}}\right)=1$. This is the main feature of the construction. The other variables $t_{i}$ and $u_{i}$ are necessary to ensure this condition. Figure 2 depicts the buildup of structures resulting from these eight properties. The states shown are necessary in a model for $\psi^{\prime}$, however there can be more states in between but those have the same assignment as one of the displayed states. The assignment for the $\forall$-quantified variables $x_{p_{1}}, \ldots, x_{p_{k}}$ is given for all states and one can see that all possible assignments are present. Assignments to the $\exists$-quantified variables are not displayed because they can differ from structure to structure. The variables $u_{i}, t_{i}$ label all states which set them to true.

For $n=0$ it holds that $\psi^{\prime}=\alpha \wedge\left(\varphi \mathrm{S} t_{0}\right)$. Since $\alpha$ satisfies the prerequisites of the claim above, there exist natural numbers $0=a_{0}<a_{1}<a_{2}<a_{3} \leq m+1$ such that

- $m-a_{1}<j \leq m-a_{0}$ implies $S, s_{j} \vDash u_{0} \wedge \overline{t_{0}}$

- $m-a_{2}<j \leq m-a_{1}$ implies $S, s_{j} \vDash \overline{u_{0}} \wedge \overline{t_{0}}$

- $m-a_{3}<j \leq m-a_{2}$ implies $S, s_{j} \vDash \overline{u_{0}} \wedge t_{0}$

The only occurring variables are $u_{0}$ and $t_{0}$ and it is easy to see that the above property of $S$ holds for both.

For the induction step assume that $n>1$ and the claim holds for $n-1$. There are two cases to consider:

Case 1: $Q_{n}=\forall$. That means

$$
\begin{aligned}
\psi^{\prime}= & \alpha \wedge \bigwedge_{i \in I_{\forall} \backslash\{n\}}\left(\left(\beta^{1}[i] \wedge \beta^{2}[i]\right) \mathrm{S} t_{0}\right) \wedge \bigwedge_{i \in I_{\exists}}\left(\left(\gamma^{1}[i] \vee \gamma^{2}[i]\right) \mathrm{S} t_{0}\right) \wedge\left(\varphi \mathrm{S} t_{0}\right) \\
& \wedge\left(\left(\beta^{1}[n] \wedge \beta^{2}[n]\right) S t_{0}\right)
\end{aligned}
$$

It follows that there are natural numbers $0=a_{0}<\cdots<a_{3\left(2^{k-1}\right)} \leq m+1$ and for every $q \in I_{\exists}$ a function $\sigma_{q}:\{0,1\}^{q-1} \rightarrow\{0,1\}$ such that $S$ fulfills the properties of the claim (note that the subformula $\left(\varphi \mathrm{S} t_{0}\right)$ is not necessary for our argument). Since $S, s_{m} \vDash\left(\beta^{1}[n] \wedge \beta^{2}[n]\right) S t_{0}$ and for $m-a_{3\left(2^{k-1}\right)}<j \leq m$ it holds that $S, s_{j} \vDash t_{0}$ if and only if $j \leq m-a_{3\left(2^{k-1}\right)-1}$, we have $S, s_{j} \vDash \beta^{1}[n] \wedge \beta^{2}[n]$ for every $m-a_{3\left(2^{k-1}\right)-1}<j \leq m$. Let $i=c \cdot 3$ for some $c \in \mathbb{N}$, then it holds that $m-a_{i+1}<j \leq m-a_{i}$ implies $S, s_{j} \vDash u_{n-1}$ which means that for these 


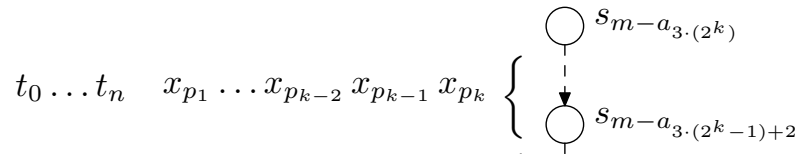

$$
\begin{aligned}
& x_{p_{1}} \ldots x_{p_{k-2}} x_{p_{k-1}} x_{p_{k}}\left\{\begin{array}{c}
1 \\
S_{m-a_{3 \cdot\left(2^{k}-1\right)+1}} s
\end{array}\right. \\
& u_{p_{k}} \ldots u_{n} \quad x_{p_{1}} \ldots x_{p_{k-2}} x_{p_{k-1}} x_{p_{k}}\left\{\begin{array}{l}
1 \\
s_{m-a_{3 \cdot\left(2^{k}-1\right)}} s
\end{array}\right. \\
& t_{p_{k}} \ldots t_{n} \quad x_{p_{1}} \ldots x_{p_{k-2}} x_{p_{k-1}} \overline{x_{p_{k}}}\left\{\begin{array}{l}
1 \\
s_{m-a_{3 \cdot\left(2^{k}-2\right)+2}} s
\end{array}\right. \\
& x_{p_{1}} \ldots x_{p_{k-2}} x_{p_{k-1}} \overline{x_{p_{k}}}\left\{\begin{array}{l}
1 \\
\underbrace{}_{m-a_{3 \cdot\left(2^{k}-2\right)+1}} s
\end{array}\right.
\end{aligned}
$$

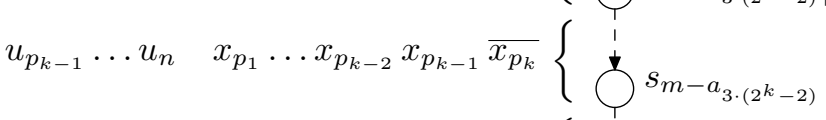

$$
\begin{aligned}
& t_{p_{k-1}} \ldots t_{n} \quad x_{p_{1}} \ldots x_{p_{k-2}} \overline{x_{p_{k-1}}} x_{p_{k}}\left\{\begin{array}{l}
1 \\
s_{m-a_{3 \cdot\left(2^{k}-3\right)+2}}
\end{array}\right. \\
& x_{p_{1}} \ldots x_{p_{k-2}} \overline{x_{p_{k-1}}} x_{p_{k}}\left\{\begin{array}{l}
1 \\
\underbrace{}_{m-a_{3 \cdot\left(2^{k}-3\right)+1}} s
\end{array}\right. \\
& u_{p_{k}} \ldots u_{n} \quad x_{p_{1}} \ldots x_{p_{k-2}} \overline{x_{p_{k-1}}} x_{p_{k}}\left\{\begin{array}{l}
1 \\
s_{m-a_{3 \cdot\left(2^{k}-3\right)}}
\end{array}\right. \\
& t_{p_{k}} \ldots t_{n} \quad x_{p_{1}} \ldots x_{p_{k-2}} \overline{x_{p_{k-1}}} \overline{x_{p_{k}}}\{\underbrace{}_{m-a_{3 \cdot\left(2^{k}-4\right)+2}} \\
& x_{p_{1}} \ldots x_{p_{k-2}} \overline{x_{p_{k-1}}} \overline{x_{p_{k}}}\left\{\begin{array}{c}
1 \\
S_{m-a_{3 \cdot\left(2^{k}-4\right)+1}} s
\end{array}\right. \\
& u_{p_{k-2}} \ldots u_{n} \quad x_{p_{1}} \ldots x_{p_{k-2}} \overline{x_{p_{k-1}}} \overline{x_{p_{k}}}\left\{\begin{array}{l}
1 \\
s_{\left.m-a_{3 \cdot(2}-4\right)}
\end{array}\right. \\
& t_{p_{k-1} \ldots t_{n}} \overline{x_{p_{1}}} \ldots \overline{x_{p_{k-2}}} \overline{x_{p_{k-1}}} x_{p_{k}}\left\{\begin{array}{c}
\bigcirc_{m-a_{6}} \\
1 \\
\bigcup_{m-a_{5}} s
\end{array}\right. \\
& \overline{x_{p_{1}}} \ldots \overline{x_{p_{k-2}}} \overline{x_{p_{k-1}}} x_{p_{k}}\left\{\begin{array}{c}
1 \\
\bigcirc_{m-a_{4}}
\end{array}\right. \\
& u_{p_{k}} \ldots u_{n} \quad \overline{x_{p_{1}}} \ldots \overline{x_{p_{k-2}}} \overline{x_{p_{k-1}}} x_{p_{k}}\left\{\begin{array}{l}
1 \\
s_{m-a_{3}}
\end{array}\right. \\
& t_{p_{k}} \ldots t_{n} \quad \overline{x_{p_{1}}} \ldots \overline{x_{p_{k-2}}} \overline{x_{p_{k-1}}} \overline{x_{p_{k}}}\left\{\begin{array}{l}
1 \\
s_{m-a_{2}}
\end{array}\right. \\
& \overline{x_{p_{1}}} \ldots \overline{x_{p_{k-2}}} \overline{x_{p_{k-1}}} \overline{x_{p_{k}}}\left\{\begin{array}{c}
1 \\
\bigcirc_{m-a_{1}}
\end{array}\right. \\
& u_{0} \ldots u_{n} \quad \overline{x_{p_{1}}} \ldots \overline{x_{p_{k-2}}} \overline{x_{p_{k-1}}} \overline{x_{p_{k}}}\left\{\begin{array}{c}
1 \\
\vdots s_{m-a_{0}}
\end{array}\right.
\end{aligned}
$$

Figure 2: Structure of models of $\psi^{\prime}$ in the proof of Theorem 3.3 
states $s_{j}$ it holds that $S, s_{j} \vDash u_{n-1} \wedge \overline{t_{n-1}} \wedge u_{n} \wedge \overline{t_{n}} \wedge x_{n}$. Due to our proposition there are natural numbers $0=b_{0}^{i}<b_{1}^{i}<\cdots<b_{6}^{i} \leq a_{i}+1$ such that

- $a_{i}-b_{1}^{i}<j \leq a_{i}-b_{0}^{i}$ implies $S, s_{j} \vDash u_{n-1} \wedge \overline{t_{n-1}} \wedge u_{n} \wedge \overline{t_{n}} \wedge \overline{x_{n}}$

- $a_{i}-b_{2}^{i}<j \leq a_{i}-b_{1}^{i}$ implies $S, s_{j} \vDash \overline{u_{n-1}} \wedge \overline{t_{n-1}} \wedge \overline{u_{n}} \wedge \overline{t_{n}} \wedge \overline{x_{n}}$

- $a_{i}-b_{3}^{i}<j \leq a_{i}-b_{2}^{i}$ implies $S, s_{j} \vDash \overline{u_{n-1}} \wedge \overline{t_{n-1}} \wedge \overline{u_{n}} \wedge t_{n} \wedge \overline{x_{n}}$

- $a_{i}-b_{4}^{i}<j \leq a_{i}-b_{3}^{i}$ implies $S, s_{j} \vDash \overline{u_{n-1}} \wedge \overline{t_{n-1}} \wedge u_{n} \wedge \overline{t_{n}} \wedge x_{n}$

- $a_{i}-b_{5}^{i}<j \leq a_{i}-b_{4}^{i}$ implies $S, s_{j} \vDash \overline{u_{n-1}} \wedge \overline{t_{n-1}} \wedge \overline{u_{n}} \wedge \overline{t_{n}} \wedge x_{n}$

- $a_{i}-b_{6}^{i}<j \leq a_{i}-b_{5}^{i}$ implies $S, s_{j} \vDash \overline{u_{n-1}} \wedge t_{n-1} \wedge \overline{u_{n}} \wedge t_{n} \wedge x_{n}$

The nearest state before $s_{m-a_{i}}$ that satisfies $\overline{u_{n-1}}$ is $s_{m-a_{i+1}}$ and the nearest state before $s_{m-a_{i}}$ that satisfies $t_{n-1}$ is $s_{m-a_{i+2}}$, therefore it holds that $b_{1}^{i}=a_{i+1}-a_{i}$ and $b_{5}^{i}=a_{i+2}-a_{i}$. By denoting $b_{j}^{i}+a_{i}$ with $c_{2 i+j}$ we define natural numbers $c_{0}, \ldots, c_{3\left(2^{k}\right)}$ for which it can be verified that they fulfill the claim.

Case 2: $Q_{n}=\exists$. In this case we have

$$
\begin{aligned}
\psi^{\prime}= & \alpha \wedge \bigwedge_{i \in I_{\forall}}\left(\left(\beta^{1}[i] \wedge \beta^{2}[i]\right) \mathrm{S} t_{0}\right) \wedge \bigwedge_{i \in I_{\exists} \backslash\{n\}}\left(\left(\gamma^{1}[i] \vee \gamma^{2}[i]\right) \mathrm{S} t_{0}\right) \wedge\left(\varphi \mathrm{S} t_{0}\right) \\
& \wedge\left(\left(\gamma^{1}[n] \vee \gamma^{2}[n]\right) S t_{0}\right) .
\end{aligned}
$$

Because of the induction hypothesis there are natural numbers $0=a_{0}<a_{1}<\cdots<a_{3\left(2^{k}\right)} \leq$ $m+1$ such that the required properties are satisfied. Analogously to the first case $S, s_{j} \vDash$ $\gamma^{1}[n] \vee \gamma^{2}[n]$ is true for every $m-a_{3\left(2^{k}\right)}<j \leq m$. Let $i=c \cdot 3$, then for $m-a_{i+1}<j \leq m-a_{i}$ it holds that $S, s_{j} \vDash u_{n-1} \wedge \overline{t_{n-1}} \wedge u_{n} \wedge \overline{t_{n}} \wedge x_{n}$ or $S, s_{j} \vDash u_{n-1} \wedge \overline{t_{n-1}} \wedge u_{n} \wedge \overline{t_{n}} \wedge \overline{x_{n}}$, because $S, s_{j} \vDash u_{n-1}$. For $m-a_{i+2}<j \leq m-a_{i+1}$ we have that $S, s_{j} \vDash \overline{u_{n-1}} \wedge \overline{t_{n-1}} \wedge \overline{u_{n}} \wedge \overline{t_{n}} \wedge x_{n}$ or $S, s_{j} \vDash \overline{u_{i-n}} \wedge \overline{t_{i-n}} \wedge \overline{u_{n}} \wedge \overline{t_{n}} \wedge \overline{x_{n}}$ and for $m-a_{i+3}<j \leq m-a_{i+2}$ it must hold $S, s_{j} \vDash \overline{u_{n-1}} \wedge t_{n-1} \wedge \overline{u_{n}} \wedge t_{n} \wedge x_{n}$ or $S, s_{j} \vDash \overline{u_{n-1}} \wedge t_{n-1} \wedge \overline{u_{n}} \wedge t_{n} \wedge \overline{x_{n}}$. If $S, s_{a_{i}} \vDash \gamma^{1}[n]$, then in all these states $\overline{x_{n}}$ is satisfied; if $S, s_{a_{i}} \vDash \gamma^{2}[n]$, then $x_{n}$ is. Therefore with $\sigma_{n}$ defined by $\sigma_{n}\left(d_{1}, \ldots, d_{n-1}\right)=1$ if and only if $S, s_{3\left(d_{1} 2^{n-2}+\cdots+d_{n-1} 2^{0}\right)} \vDash \gamma^{2}[n]$, the induction is complete, because the binary numbers correspond to the assignments to the $\forall$-quantified variables.

Note that for a structure that satisfies $\psi^{\prime}$ with the above notation, $S, s_{j} \vDash \varphi$ holds for every $m-a_{3\left(2^{k}\right)}<j \leq m$, since $\varphi \mathrm{S} t_{0}$ is a conjunct of $\psi^{\prime}$.

Now assume that $\psi^{\prime}$ is satisfiable in a state $s_{m}$ of a structure $S$. This is if and only if for every $q \in I_{\exists}$ there is a function $\sigma_{q}:\{0,1\}^{q-1} \rightarrow\{0,1\}$ such that $S$ fulfills the above property. Hence each possible assignment $J$ to the $\forall$-quantified variables $\left\{x_{p_{1}}, \ldots, x_{p_{k}}\right\}$ can be extended to an assignment to $\left\{x_{1}, \ldots, x_{n}\right\}$ by $J\left(x_{q_{i}}\right)=\sigma_{q_{i}}\left(J\left(x_{1}\right), \ldots, J\left(x_{q_{i}-1}\right)\right)$ which is equivalent to the validity of $\psi$. We can prove PSPACE-hardness for $\operatorname{SAT}(\{\mathrm{U}\}, B)$ with an analogous construction.

In the following, we use the result from Lewis [Lew79] and the previously established upper bounds to obtain NP-completeness results:

Proposition 3.4. Let $B$ be a finite set of Boolean functions such that $\mathrm{S}_{1} \subseteq[B]$. Then $\operatorname{SAT}(\{\mathrm{F}\}, B), \operatorname{SAT}(\{\mathrm{G}\}, B), \operatorname{SAT}(\{\mathrm{F}, \mathrm{G}\}, B)$, and $\mathrm{SAT}(\{\mathrm{X}\}, B)$ are NP-complete.

Proof. Trivially, it holds that $\operatorname{SAT}(\emptyset, B) \leq_{m}^{\log } \operatorname{SAT}(M, B)$ for each set $M$ of temporal operators, and $\operatorname{SAT}(\emptyset, B)$ is NP-complete due to [Lew79]. The upper bound follows from Theorem 2.111 and Lemma 3.1. 
3.2. Polynomial time results. The following theorem shows that for some sets $B$ of Boolean functions, there is a satisfying model for every temporal $B$-formula over any set of temporal operators. These are the cases where $B \subseteq \mathrm{R}_{1}$, or $B \subseteq \mathrm{D}$. In the first case, every propositional formula over these operators is satisfied by the assignment giving the value 1 to all appearing variables. In the second case, every propositional $B$-formula describes a self-dual function. For such a formula it holds in particular that if it is not satisfied by the all-zero assignment, then it is satisfied by the all-one assignment. Hence, such formulae are always satisfiable. It is easy to see that this is also true for temporal formulae involving these propositional operators.

\section{Theorem 3.5.}

(1) Let $B$ be a finite subset of $\mathrm{R}_{1}$. Then every formula $\varphi$ from $\mathrm{L}(\{\mathrm{F}, \mathrm{G}, \mathrm{X}, \mathrm{U}, \mathrm{S}\}, B)$ is satisfiable.

(2) Let $B$ be a finite subset of $\mathrm{D}$. Then every formula $\varphi$ from $\mathrm{L}(\{\mathrm{F}, \mathrm{G}, \mathrm{X}, \mathrm{U}, \mathrm{S}\}, B)$ is satisfiable.

Proof.

(1) Since $\mathrm{R}_{1}$ is the class of 1-reproducing Boolean functions, any $\psi \in \mathrm{R}_{1}$ is true under the assignment that makes every propositional variable in $\psi$ true. If we apply this fact to formulae $\varphi \in \mathrm{L}(\{\mathrm{F}, \mathrm{G}, \mathrm{X}, \mathrm{U}, \mathrm{S}\}, B)$, then it is easy to see that any such formula $\varphi$ is true in every state of a structure $S_{\varphi}$ where the assignment of every state is $V_{\varphi}$.

(2) We show by induction on the operators that this holds for all formulae. Let $S^{1}\left(S^{0}\right)$ denote the structure where the assignment of every state is $V_{\varphi}\left(\emptyset\right.$, resp.) and let $s^{1}\left(s^{0}\right.$, resp.) be the first state. We claim that $\varphi \in \mathrm{L}(\{\mathrm{F}, \mathrm{G}, \mathrm{X}, \mathrm{U}, \mathrm{S}\}, B)$ is satisfied by $S^{1}$ iff $\varphi$ is not satisfied by $S^{0}$. If $\varphi$ is purely propositional the claim holds trivially. We now have to look at the following cases:

- $\varphi=\mathrm{F} \varphi_{1}$ : Assume the claim holds for $\varphi_{1}$. Since for all states $s$ in $S^{1}$ the submodel starting at $s$ are isomorphic, obviously $\varphi$ is satisfied by $S^{1}$ iff $\mathrm{F} \varphi$ is satisfied by $S^{1}$ and the same argument also holds for $S^{0}$. Thus $S^{0}, s^{0} \not \models \varphi$ iff $S^{1}, s^{1} \vDash \varphi$.

- $\varphi=\mathrm{G} \varphi_{1}$ : This works analogously to $\mathrm{F}$.

- $\varphi=\mathrm{X} \varphi_{1}$ : This also works analogously to $\mathrm{F}$.

- $\varphi=\varphi_{1} \cup \varphi_{2}$ : Assume the claim holds for $\varphi_{2}$. Then $S^{0}, s^{0} \not \models \varphi$ iff $S^{0}, s^{0} \not \models \varphi_{2}$ iff $S^{1}, s^{1} \vDash \varphi_{2}$ iff $S^{1}, s^{1} \vDash \varphi$.

- $\varphi=\varphi_{1} \mathrm{~S} \varphi_{2}$ : This works analogously to $\mathrm{U}$.

- $\varphi=f\left(\varphi_{1}, \ldots, \varphi_{n}\right)$, such that $f$ is a self-dual function from $B$ : Assume the claim holds for $\varphi_{i}, 1 \leq i \leq n$, i.e., $S^{1}, s^{1} \vDash \varphi_{i}$ iff $S^{0}, s^{0} \not \models \varphi_{i}$. Then $S^{1}, s^{1} \not \models f\left(\varphi_{1}, \ldots, \varphi_{n}\right)$ implies $S^{0}, s^{0} \vDash f\left(\varphi_{1}, \ldots, \varphi_{n}\right)$ and $S^{1}, s^{1} \vDash f\left(\varphi_{1}, \ldots, \varphi_{n}\right)$ implies $S^{0}, s^{0} \not \models f\left(\varphi_{1}, \ldots, \varphi_{n}\right)$.

The following two theorems prove that satisfiability for formulae with any combination of modal operators, but only very restricted Boolean operators (i.e., negation and constants in the first case and only disjunction, conjunction, and constants in the second case), is always easy to decide.

Theorem 3.6. Let $B$ be a finite subset of $\mathrm{N}$. Then $\mathrm{SAT}(\{\mathrm{F}, \mathrm{G}, \mathrm{X}, \mathrm{U}, \mathrm{S}\}, B)$ can be decided in polynomial time.

Proof. We give a recursive polynomial-time algorithm deciding the following question: Given a formula $\varphi$ built from propositional negation, constants, variables and arbitrary temporal operators, which of the following three cases occurs: $\varphi$ is unsatisfiable, $\varphi$ is a tautology, or $\varphi$ is not equivalent to a constant function. We also show that in the latter case, $\varphi$ is 
equivalent to a formula using only the above operators in which no constant appears. We will call these formulae temporal $\neg$-formulae.

We give inductive criteria for these cases. Obviously, a constant $c$ is constant, and a variable is not, and can be written in the way defined above. The formula $\neg \varphi$ is equivalent to the constant $c$ if and only if $\varphi$ is equivalent to $\neg c$, otherwise it is equivalent to a temporal -formula. If $\varphi=\mathrm{F} \varphi_{1}, \varphi=\mathrm{G} \varphi_{1}$, or $\varphi=\mathrm{X} \varphi_{1}$, then $\varphi$ is equivalent to a constant $c$ if and only if $\varphi_{1}$ is equivalent to $c$ : Obviously $\mathrm{F} c \equiv \mathrm{G} c \equiv \mathrm{X} c \equiv c$ for a constant. On the other hand, if $\varphi_{1}$ is not equivalent to a constant, then due to induction, it is equivalent to a temporal $\neg$-formula. Hence, $F \varphi_{1}, G \varphi_{1}$ and $X \varphi_{1}$ are equivalent to temporal $\neg$-formulae as well, and due to the proof of Theorem 3.5, 2, these formulae are not equivalent to constants. Hence, if $\varphi_{1}$ is not equivalent to a constant, then $\varphi$ is not equivalent to a constant either, and can be written as a temporal $\neg$-formula.

Now, let $\varphi=\varphi_{1} \cup \varphi_{2}$. If $\varphi_{2}$ is a tautology, i.e., equivalent to the constant 1 , then, by the definition of $U, \varphi$ is a tautology as well. Similarly, if $\varphi_{2}$ is equivalent to the constant 0 , then so is $\varphi$. Now assume that $\varphi_{2}$ is not constant. Then, by induction, $\varphi_{2}$ is equivalent to a temporal $\neg$-formula. If $\varphi_{1}$ is equivalent to the constant 0 , then $\varphi_{1} \cup \varphi_{2}$ is equivalent to $\varphi_{2}$, and if $\varphi_{1}$ is equivalent to 1 , then $\varphi_{1} \mathrm{U} \varphi_{2}$ is equivalent to $\mathrm{F} \varphi_{2}$. If $\varphi_{1}$ is not equivalent to a constant, then, by induction, it can be written as a temporal $\neg$-formula, and obviously, this also holds for $\varphi_{1} \cup \varphi_{2}$. Again due to the proof of Theorem 3.5, 2, it follows that the entire formula $\varphi$ is not equivalent to a constant.

For the operator $S$, a similar argument can be made: Consider the formula $\varphi_{1} S \varphi_{2}$. If $\varphi_{2}$ is a constant, then obviously the formula $\varphi_{1} S \varphi_{2}$ is equivalent to the same constant. If $\varphi_{1}$ is the constant 0 , then $\varphi_{1} \mathrm{~S} \varphi_{2}$ is equivalent to $\varphi_{2}$, and if $\varphi_{1}$ is the constant 1 , then $\varphi_{1} \mathrm{~S} \varphi_{2}$ is equivalent to " $\varphi_{2}$ was true at one point in the past." If $\varphi_{2}$ is not a constant, then this is equivalent to $\neg \varphi_{2} \mathrm{~S} \varphi_{2}$, and thus this can be written as a temporal $\neg$-formula as well. As above, this formula is not equivalent to a constant. Now if both $\varphi_{1}$ and $\varphi_{2}$ are not equivalent to a constant function, then, by induction, both can be written as temporal $\neg$-formulae, and then $\varphi_{1} \mathrm{~S} \varphi_{2}$ can be written as such a formula as well. In particular, with another application of the proof for Theorem 3.5]2, $\varphi_{1} \mathrm{~S} \varphi_{2}$ is not equivalent to a constant.

This gives us a recursive algorithm deciding whether $\varphi$ is a constant, and if it is, which constant is equivalent to $\varphi$. The polynomial-time computable function $A_{N}$ is defined as follows: On input $\varphi, A_{N}(\varphi)=c \in\{0,1\}$ if $\varphi$ is equivalent to the constant $c$, and $A_{N}(\varphi)$ is the symbol NOCONSTANT if $\varphi$ is not equivalent to a constant.

The function can be computed as follows: $A_{N}(c)$ is defined as $c$. For a variable $x$, $A_{N}(x)$ is the symbol nOCONSTANT. On input $\mathrm{X} \varphi, \mathrm{G} \varphi$, or $\mathrm{F} \varphi$, the algorithm returns $A_{N}(\varphi)$. On input $\varphi_{1} \mathrm{U} \varphi_{2}$, if $\varphi_{2}$ is a constant $c$, then $A_{N}\left(\varphi_{1} \cup \varphi_{2}\right)=c$. Otherwise, if $\varphi_{1}$ is equivalent to 0 , then return $A_{N}\left(\varphi_{2}\right)$, and if $\varphi_{1}$ is equivalent to 1 , return $A_{N}\left(\mathrm{~F} \varphi_{2}\right)$. If neither $\varphi_{1}$ nor $\varphi_{2}$ are constant, then return the symbol NOCONSTANT. Similarly, on input $\varphi_{1} \mathrm{~S} \varphi_{2}$, if $\varphi_{2}$ is a constant $c$, then $A_{N}\left(\varphi_{1} \mathrm{~S} \varphi_{2}\right)=c$. Otherwise, if $\varphi_{1}$ is the constant 0 , then $A_{N}\left(\varphi_{1} \mathrm{~S} \varphi_{2}\right)=A_{N}\left(\varphi_{2}\right)$, and if $\varphi_{1}$ is the constant 1 , and $\varphi_{2}$ is not a constant, then $A_{N}\left(\varphi_{1} S \varphi_{2}\right)$ is defined as the symbol NOCONSTANT. If $\varphi_{1}$ and $\varphi_{2}$ both are not a constant, then $A_{N}\left(\varphi_{1} S \varphi_{2}\right)$ is again defined as the symbol NOCONSTANT. The function $A_{N}$ can obviously be computed in polynomial time, since there is at most one recursive call for each operator symbol in $\varphi$.

By the argument above, this algorithm correctly determines if $\varphi$ is equivalent to the constant 0 or the constant 1 . In particular, it determines if a given formula is satisfiable.

Theorem 3.7. Let $B$ be a finite subset of $\mathrm{M}$. Then $\mathrm{SAT}(\{\mathrm{F}, \mathrm{G}, \mathrm{X}, \mathrm{U}, \mathrm{S}\}, B)$ can be decided in polynomial time. 
Proof. Remember that $\mathrm{M}$ is the clone of all monotone functions. Let $\varphi$ be an arbitrary formula from $\mathrm{L}(\{\mathrm{F}, \mathrm{G}, \mathrm{X}, \mathrm{U}, \mathrm{S}\}, B)$. The following algorithm decides whether $\varphi$ is satisfiable.

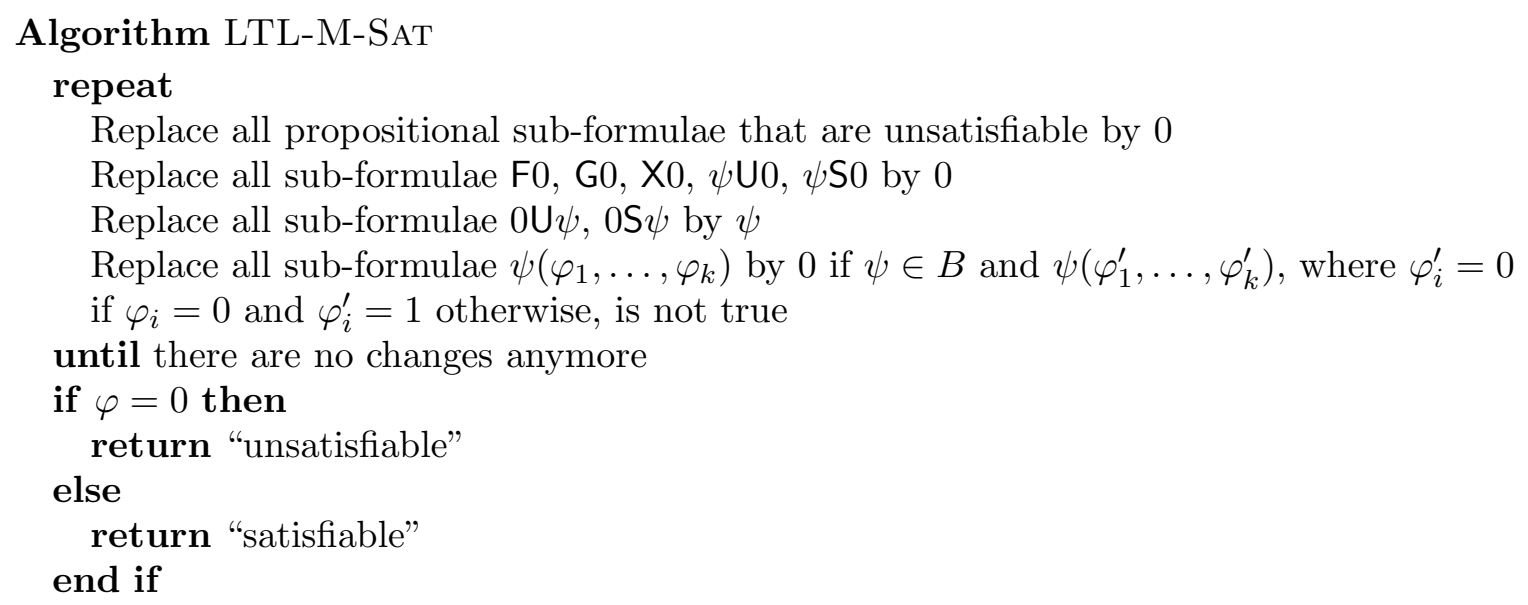

Since checking satisfiability of propositional $B$-formulae is in P (a $B$-formula $\varphi$ is satisfiable iff $\varphi(1, \ldots, 1)=1$ ) and there are at most as many replacements as there are operators in $\varphi$, LTL-M-SAT runs in polynomial time.

We prove that LTL-M-SAT is correct. If $\varphi$ is satisfiable, then LTL-M-SAT returns "satisfiable." This is because all replacements in LTL-M-SAT do not affect satisfiability, so it follows that every formula LTL-M-SAT decides to be unsatisfiable is unsatisfiable. For the converse direction, let $\varphi \in \mathrm{L}(\{\mathrm{F}, \mathrm{G}, \mathrm{X}, \mathrm{U}, \mathrm{S}\}, B)$ be such that LTL-M-SAT returns "satisfiable" and let $\varphi^{\prime}$ be the formula generated by LTL-M-SAT in its REPEAT loop. We show by induction on the structure of $\varphi$ that $S, s_{0} \vDash \varphi$, where $S=\left(s, V_{\varphi}, \xi\right)$ is the structure in which every variable is true in every state, i.e., $\xi\left(s_{i}\right)=V_{\varphi}$ for every $i \in \mathbb{N}$.

(1) If $\varphi$ is a variable, it is satisfied in $S, s_{0}$ trivially.

(2) If $\varphi=\mathrm{F} \psi$ for a formula $\psi \in \mathrm{L}(\{\mathrm{F}, \mathrm{G}, \mathrm{X}, \mathrm{U}, \mathrm{S}\}, B)$, let $\psi^{\prime}$ be the formula generated in the REPEAT loop when performing LTL-M-SAT on $\psi$. Assume that $\psi^{\prime}=0$. Since every subformula replaced in $\psi$ by LTL-M-SAT will be replaced in $\varphi$, too, it holds that $\mathrm{F} \psi$ will be replaced by F0 and that will be replaced by 0 . It follows that $\varphi^{\prime}=0$, but then LTL-M-SAT would return "unsatisfiable." Thus, $\psi^{\prime} \neq 0$, that means LTL-M-SAT returns "satisfiable" when performed on $\psi$. By induction it follows that $S, s_{0} \vDash \psi$ and therefore $S, s_{0} \vDash \varphi$ holds as well.

(3) If $\varphi=\mathrm{G} \psi$ for a formula $\psi \in \mathrm{L}(\{\mathrm{F}, \mathrm{G}, \mathrm{X}, \mathrm{U}, \mathrm{S}\}, B)$, we can use exactly the same arguments as in 2 .

(4) If $\varphi=\mathrm{X} \psi$ for a formula $\psi \in \mathrm{L}(\{\mathrm{F}, \mathrm{G}, \mathrm{X}, \mathrm{U}, \mathrm{S}\}, B)$, we can use the same arguments as in 2.

(5) If $\varphi=\psi_{1} \mathrm{U} \psi_{2}$ for formulae $\psi_{1}, \psi_{2} \in \mathrm{L}(\{\mathrm{F}, \mathrm{G}, \mathrm{X}, \mathrm{U}, \mathrm{S}\}, B)$, we have that $\psi_{2}$ cannot be replaced by 0 (otherwise $\varphi$ would be replaced by 0 and LTL-M-SAT would return "unsatisfiable"). So by induction it follows that $S, s_{0} \vDash \psi_{2}$. Hence, it holds that $S, s_{0} \vDash \varphi$ as well.

(6) If $\varphi=\psi_{1} \mathrm{~S} \psi_{2}$ for formulae $\psi_{1}, \psi_{2} \in \mathrm{L}(\{\mathrm{F}, \mathrm{G}, \mathrm{X}, \mathrm{U}, \mathrm{S}\}, B)$, we can use the same arguments as for 5 .

(7) If $\varphi=\psi\left(\varphi_{1}, \ldots, \varphi_{k}\right)$ for formulae $\psi \in B$ and $\varphi_{i} \in \mathrm{L}(\{\mathrm{F}, \mathrm{G}, \mathrm{X}, \mathrm{U}, \mathrm{S}\}, B)$, for all $i=$ $1, \ldots, k$, let $\varphi_{1}^{\prime}, \ldots, \varphi_{k}^{\prime}$ be the replacements of $\varphi_{1}, \ldots, \varphi_{k}$. By induction it follows that 
$S, s_{0} \vDash \varphi_{i}$ if and only if $\varphi_{i}^{\prime} \neq 0$ for any $i \in\{1, \ldots, k\}$. Since $\varphi^{\prime} \neq 0$ and because of the last replacement rule, $S, s_{0} \vDash \varphi$.

Finally, we show that satisfiability for formulae that have $\mathrm{X}$ as a modal operator and the xor function $\oplus$ as a propositional operator is in $\mathrm{P}$. This is true because functions described by these formulae have a high degree of symmetry.

Theorem 3.8. Let $B$ be a finite subset of $\mathrm{L}$. Then $\mathrm{SAT}(\{\mathrm{X}\}, B)$ can be decided in polynomial time.

Proof. First observe that any function from $\mathrm{L}$ is of the form $f\left(x_{1}, \ldots, x_{n}\right)=x_{i_{1}} \oplus \cdots \oplus x_{i_{k}} \oplus c$, where the $x_{i_{j}}$ are pairwise different variables from the set $\left\{x_{1}, \ldots, x_{n}\right\}$, and $c$ is either 0 or 1 . Therefore, it is obvious that temporal $B$-formulae can be rewritten using only the connectors $\oplus$ and the constant 1 (the 0 can be omitted in the representation above). Hence, we can assume that the set $B$ contains only the functions $\oplus$ and 1 . Now observe that any formula $\varphi$ from $\mathrm{L}(\{\mathrm{X}\},\{\oplus, 1\})$ can be written as

$$
\varphi=\mathrm{X} \psi_{1} \oplus \cdots \oplus \mathrm{X} \psi_{k} \oplus \psi,
$$

where $\psi$ is a propositional formula. This representation can be computed in polynomial time, and we can determine in polynomial time whether $\psi$ is a constant function.

If $\psi$ is not a constant function, then $\varphi$ is satisfiable: Let $S=\left(s, V_{\varphi}, \xi\right)$ be an arbitrary structure. If $\varphi$ is not satisfied at $s_{0}$, then we can "switch over" the current truth value of $\psi$, thus achieving that one more (or one less) of the arguments of the outermost xor function becomes true. For this purpose, we change the assignment of the propositional variables at $s_{0}$ in such a way that the new assignment satisfies $\psi$ if and only if the old assignment does not. Since this change does not affect the validity of the $\mathbf{X} \psi_{i}$ parts, $\varphi$ holds at $s_{0}$ with the new assignment.

Now, if $\psi$ is constant, this trick does not work. Instead, let

$$
\varphi^{\prime}=\psi_{1} \oplus \cdots \oplus \psi_{k} .
$$

Observe that in this case $\varphi$ is satisfiable if and only if $\psi$ is the constant 0 and $\varphi^{\prime}$ is satisfiable, or if $\psi$ is the constant 1 and $\varphi^{\prime}$ is no tautology; and that $\varphi$ is a tautology if and only if $\psi$ is the constant 0 and $\varphi^{\prime}$ is a tautology, or $\psi$ is the constant 1 and $\varphi^{\prime}$ is not satisfiable. Thus we have an iterative algorithm deciding $\operatorname{SAT}(\{X\},\{\oplus, 1\})$, since for a propositional $B$-formula, these questions can be efficiently decided.

\section{Conclusion}

We have almost completely classified the computational complexity of satisfiability for LTL with respect to the sets of propositional and temporal operators permitted, see Table 3. The only case left open is the one in which only propositional operators constructed from the binary xor function (and, perhaps, constants) are allowed. This case has already turned out to be difficult to handle - and hence was left open - in BHSS06] for modal satisfiability under restricted frames classes. The difficulty here and in [BHSS06] is reflexivity, i.e., the property that the formula $\mathrm{F} \varphi$ is satisfied at some state if $\varphi$ is satisfied at the same state. This does not allow for a separate treatment of the propositional part (without temporal operators) and the remainder of a given formula.

Our results bear an interesting resemblance to the classifications obtained in Lew79 and in BHSS06. In all of these cases (except for one of the several classifications obtained 


\begin{tabular}{l|cc}
$\begin{array}{r}\text { temporal operators } \\
\text { function class } B \text { (propositional operators) }\end{array}$ & $\begin{array}{c}\{\mathrm{F}\},\{\mathrm{G}\}, \\
\{\mathrm{F}, \mathrm{G}\},\{\mathrm{X}\}\end{array}$ & $\begin{array}{c}\text { any other } \\
\text { combination }\end{array}$ \\
\hline$B \subseteq \mathrm{R}_{1}$ or $B \subseteq \mathrm{D}$ & trivial & trivial \\
$B \subseteq \mathrm{M}$ or $B \subseteq \mathrm{N}$ & in $\mathrm{P}$ & in $\mathrm{P}$ \\
$\mathrm{L}_{0}, \mathrm{~L}$ & $?$ & $?$ \\
else $\left(i . e ., B \supseteq \mathrm{S}_{1}\right)$ & $\mathrm{NP}-\mathrm{c}$. & PSPACE-c.
\end{tabular}

Table 3: Complexity results for satisfiability. The entries "trivial" denote cases in which a given formula is always satisfiable. The abbreviation "c." stands for "complete." Question marks stand for open questions.

in the latter), it turns out that sets of Boolean functions $B$ which generate a clone above $\mathrm{S}_{1}$ give rise to computationally hard problems, while other cases seem to be solvable in polynomial time. Therefore, in a precise sense, it is the function represented by the formula $x \wedge \bar{y}$ which turns problems in this context computationally intractable. These hardness results seem to indicate that $x \wedge \bar{y}$ and other functions which generate clones above $\mathrm{S}_{1}$ have properties that make computational problems hard, and this notion of hardness is to a large extent independent of the actual problem considered.

In [BMS ${ }^{+}$07, we have separated tractable and intractable cases of the model checking problem for LTL with restrictions to the propositional operators. Without such restrictions, this problem has the same complexity as satisfiability [SC85.

The results from this paper leave two open questions. Besides the unsolved xor case, it would be interesting to further classify the polynomial-time solvable cases. Further work could also examine related specification languages, such as CTL, CTL* ${ }^{*}$, or hybrid temporal languages.

\section{ACKNOWLEDGMENTS}

We thank Martin Mundhenk and the anonymous referees for helpful comments and suggestions.

\section{REFERENCES}

[BCRV03] E. Böhler, N. Creignou, S. Reith, and H. Vollmer. Playing with Boolean blocks, part I: Post's lattice with applications to complexity theory. SIGACT News, 34(4):38-52, 2003.

[BHSS06] M. Bauland, E. Hemaspaandra, H. Schnoor, and I. Schnoor. Generalized modal satisfiability. In B. Durand and W. Thomas, editors, STACS, volume 3884 of Lecture Notes in Computer Science, pages 500-511. Springer, 2006.

$\left[\mathrm{BMS}^{+} 07\right]$ M. Bauland, M. Mundhenk, T. Schneider, H. Schnoor, I. Schnoor, and H. Vollmer. The tractability of model checking for LTL: the good, the bad, and the ugly fragments. In Proceedings Methods for Modalities 5, pages 125-140. ENS Cachan, 2007. Also at CoRR http://arxiv.org/abs/0805.0498.

$\left[\mathrm{BSS}^{+} 07\right] \quad$ M. Bauland, T. Schneider, H. Schnoor, I. Schnoor, and H. Vollmer. The complexity of generalized satisfiability for linear temporal logic. In H. Seidl, editor, FoSSaCS, volume 4423 of Lecture Notes in Computer Science, pages 48-62. Springer, 2007.

[CL93] C.-C Chen and I-P. Lin. The computational complexity of satisfiability of temporal Horn formulas in propositional linear-time temporal logic. Inf. Process. Lett., 45(3):131-136, 1993. 
[Coo71] S. A. Cook. The complexity of theorem proving procedures. In Proceedings 3rd Symposium on Theory of Computing, pages 151-158. ACM Press, 1971.

[Dal00] V. Dalmau. Computational Complexity of Problems over Generalized Formulas. PhD thesis, Department de Llenguatges i Sistemes Informàtica, Universitat Politécnica de Catalunya, 2000.

[DFR00] C. Dixon, M. Fisher, and M. Reynolds. Execution and proof in a Horn-clause temporal logic. In H. Barringer, M. Fisher, D. Gabbay, and G. Gough, editors, Advances in Temporal Logic, volume 16 of Applied Logic Series, pages 413-433. Kluwer, 2000.

[DS02] S. Demri and P. Schnoebelen. The complexity of propositional linear temporal logics in simple cases. Inf. Comput., 174(1):84-103, 2002.

[EES90] E. A. Emerson, M. Evangelist, and J. Srinivasan. On the limits of efficient temporal decidability. In LICS, pages 464-475. IEEE Computer Society, 1990.

[Hal95] J. Y. Halpern. The effect of bounding the number of primitive propositions and the depth of nesting on the complexity of modal logic. Artif. Intell., 75(2):361-372, 1995.

[Hem01] E. Hemaspaandra. The complexity of poor man's logic. J. Log. Comput., 11(4):609-622, 2001.

[Lew79] H. Lewis. Satisfiability problems for propositional calculi. Mathematical Systems Theory, 13:45$53,1979$.

[Low08] G. Lowe. Specification of communicating processes: temporal logic versus refusals-based refinement. Formal Aspects of Computing, 20(3):277-294, 2008.

[Mar04] N. Markey. Past is for free: on the complexity of verifying linear temporal properties with past. Acta Informatica, 40(6-7):431-458, 2004.

[Nor05] G. Nordh. A trichotomy in the complexity of propositional circumscription. In Proceedings of the 11th International Conference on Logic for Programming, volume 3452 of Lecture Notes in Computer Science, pages 257-269. Springer Verlag, 2005.

[Pip97] N. Pippenger. Theories of Computability. Cambridge University Press, Cambridge, 1997.

[Pnu77] A. Pnueli. The temporal logic of programs. In FOCS, pages 46-57. IEEE, 1977.

[Pos41] E. Post. The two-valued iterative systems of mathematical logic. Annals of Mathematical Studies, 5:1-122, 1941.

[Rei01] S. Reith. Generalized Satisfiability Problems. PhD thesis, Fachbereich Mathematik und Informatik, Universität Würzburg, 2001.

[RV03] S. Reith and H. Vollmer. Optimal satisfiability for propositional calculi and constraint satisfaction problems. Information and Computation, 186(1):1-19, 2003.

[RW05] S. Reith and K. W. Wagner. The complexity of problems defined by Boolean circuits. In Proceedings International Conference Mathematical Foundation of Informatics, (MFI99); World Science Publishing, 2005.

[SC85] A. Sistla and E. Clarke. The complexity of propositional linear temporal logics. Journal of the ACM, 32(3):733-749, 1985.

[Sch05] H. Schnoor. The complexity of the Boolean formula value problem. Technical report, Theoretical Computer Science, University of Hannover, 2005.

[Sto77] L. Stockmeyer. The polynomial-time hierarchy. Theoretical Computer Science, 3:1-22, 1977. 